\title{
Elastic Frustration in 1D Spin-Crossover Chains: Evidence of Multi-Step Transitions and Self-Organizations of the Spin States
}

\section{Rachid TRAICHE ${ }^{1,2}$, Mouhamadou SY ${ }^{1}$ and Kamel BOUKHEDDADEN ${ }^{1 *}$}

${ }^{1}$ Groupe d'Etudes de la Matière Condensée, Université de Versailles, Université Paris-Saclay, CNRS UMR 8635, 45 Avenue des Etats Unis 78035 Versailles

${ }^{2}$ Université Hassiba Ben Bouali-Chlef, Hay Essalem route nationale $\mathrm{N}^{\circ}$ 19, Chlef 02000, Algeria.

*corresponding authors: kamel.bouheddaden@uvsq.fr

\begin{abstract}
We consider a 1D elastic spin-crossover (SCO) chain in which each site may be in the low-spin or in the high-spin (HS) state. The sites interact elastically through a harmonic coupling and the local equilibrium distances depend on the spin states of the interacting sites. The Hamiltonian of the system is solved by Monte Carlo method running on the spin states and the atomic displacements. By considering the existence of an elastic frustration between the equilibrium distances of the nearest-neighbors and the next-nearest-neighbors, we succeeded, to highlight a number of original behaviors of the thermal dependence of the high-spin fraction, like multi-step transitions, incomplete spin transitions, emergence of self-organized structures and reentrant spin transitions, by adjusting only one control parameter. The obtained results allow understanding several experimental data of 1D spin-crossover materials which seem to be model systems for elastic frustration.
\end{abstract}

\section{Introduction}

The magnetic spin change associated with the spin crossover (SCO) phenomenon represents a new paradigm of molecular bistability which emerges at the macroscopic scale, offering potential applications in the development of new generations of electronic devices such as non-volatile memories, molecular sensors, displays and reversible switches ${ }^{1-5}$. In particular, Iron (II)-based spin-crossover materials ${ }^{1,3,6-12}$ are fascinating objects, due to their labile electronic character which confers them the interesting property to convert from the diamagnetic low-spin (LS, $e_{g}^{0} t_{2 g}^{6}$, 
$S=0$ ) to a paramagnetic high-spin (HS, $e_{g}^{2} t_{2 g}^{4}, S=2$ ) states, upon change of temperature. This feature is only possible when the energy gap between the HS and the LS states is of the order of magnitude of the thermal energy, which is possible when the central Fe-atom is surrounded by Nitrogen atoms in octahedral symmetry. However, the complete character of the transition between the LS and the HS states, even in weak-cooperative systems, cannot be understood without invoking the crucial role of the entropy difference between the LS and HS states. This entropy difference, originating from the large change in electronic and vibrational properties upon spin transition constitutes the driving force of this phenomenon. It is well known that the change in the electronic configuration of $\mathrm{Fe}$, subsequent to the internal redistribution of the charges between $e_{g}^{0}$ and $t_{2 g}$ orbitals, which are strongly coupled to the structure, significantly affects the coordination sphere of the metal, leading to a bigger volume of the molecule in the HS state. This local molecular volume change is delocalized over several unit cells through elastic interactions (which are known for their long-range nature) which propagate at the velocity of sound in the solid, leading at the transition temperature to a macroscopic volume change of $\sim 3 \%$, while the change of the Fe-ligand distances is almost $10 \%^{5,13-15}$, while the velocity of the HS-LS interfaces is around 4-5 $\mu \mathrm{m} / \mathrm{s}^{16}$. In solid state, the LS to HS conversions give rise to a rich variety of behaviours, going from (i) a gradual spin-transition transition, to (ii) sharp first-order transitions, (iii) incomplete transitions and (iv) multi-step transitions. It is important to mention that these types of transitions belong to the same class of molecular materials. For example, starting from a material exhibiting a first-order transition with hysteresis, and by changing the nature of the ligand or that of the anion $\left(\mathrm{BF}_{4}, \mathrm{PF}_{6}, \ldots\right)$ in some crystals leads to drastic changes like, the appearance of a plateau along the LS to HS transition, or the presence of an incomplete phase transition ${ }^{17}$. The reason for the sensitivity of the SCO materials to the steric effects (size of the ligands and anions) and their degrees of freedom (rotations, vibrations) along the thermal cycling is due to the important role of the elastic interactions in the SCO phenomenon which takes place cooperatively through the delocalization and interference between the local volume changes at the molecular level. As the elastic interactions propagate through the elastic medium, the nature, the mass, and the sterically hindered constituents play a major role in the resulting nucleation and growth modes of the SCO transition phenomenon. Several examples of two-steps transitions have been reported in literature. Sometimes it is due to competing short- and long-range interactions, as reported in the case of $\left[\mathrm{Fe}(2-\mathrm{pic})_{3}\right] \mathrm{Cl}_{2} \cdot \mathrm{EtOH}^{18}$. On the other hand, SCO lattices formed by interacting dinuclear molecules 
may lead to two-step SCO too. In most of the cases, the transition of the first SCO center induces a distortion that changes the ligand field of the second Fe center, which then makes the transition at a different temperature, due to an elastic frustration. The two-step transition can be also accompanied by a symmetry breaking ${ }^{19-23}$, as well as the presence of a self-organization of the spin state in the plateau region. Moreover, quite recently, some materials have been found to exhibit several steps ${ }^{21,24-25}$ at the transition, among them the case of the "Devil's Staircase"22 is a very instructive example of the complexity generated by elastic interactions inside the SCO materials. Last but not least, several two-step transitions have also reported in 1D SCO materials 26-28 REF, which confirms the general character of elastic frustration in these molecular systems.

The case of multi-step transitions is challenging and most of the theoretical descriptions were based on Ising-like models ${ }^{29-32}$ in which antiferromagnetic-like interactions are introduced in the phenomenological way. However, even though the multi-step transition is an emergence property which comes out from antagonist or competing elastic interactions inside the solid: that is the concept of elastic frustration, expressing the existence of antagonist steric effects that we introduced recently in ${ }^{19}$. There, we studied the case of a $2 \mathrm{D}$ elastic lattice that mimics a $2 \mathrm{D}$ spincrossover solid in which we have considered the existence of an energetic competition between the elastic energy along the lattice parameter direction and the diagonals. We have demonstrated that according to the rate of elastic frustration misfit between these two directions, almost all previously quoted experimental features in 2D systems can be produced, however, the case of 1D systems was not yet considered. So, based on this first success, we extend here our theory to study the case of frustrated and non-frustrated elastic 1D systems, using both Monte Carlo and analytical methods.

In this contribution, we exclusively investigate the thermodynamic properties of an isolated one dimensional elastically-frustrated SCO chain. The more general case of interacting 1D chains will be considered in a further work. The elastic frustration is introduced through the existence of antagonist nearest- and next-nearest-neighbors equilibrium distances subsequent to the appearance of LS species in the lattice on cooling, while the HS state is chosen as frustration-free by construction. In the material, the elastic frustration may be caused by the existence of direct contacts between next-nearest neighbours SCO atoms via their ligands, or due to indirect interactions through adjacent chains which imposes additional constraints which oppose to the 
degrees of freedom contacts (expansion or contraction) of the nearest-neighbours SCO atoms. Here, we modelled this additional interaction through a direct next-nearest neighbour elastic coupling between the SCO atoms that generates elastic frustration with the interaction between immediate surrounding atoms. We first demonstrate, using Monte Carlo (MC) simulations, that an isolated elastic chain of interacting SCO atoms leads easily to kinetic hysteretic first-order transitions, in good agreement with previous MC simulations performed on a different type of elastic model ${ }^{33}$. Second, the introduction of an elastic frustration leads to a rich variety of thermal behaviour of the HS fraction, among which the famous multi-step 11,21-24, 34-38 and incomplete transitions which lead to residual HS fractions at low-temperature, as well as re-entrant phase transitions ${ }^{39}$ based on size effect changes. In addition, we observed the presence of clear spatial self-organizations modes of the HS and LS states in the plateau regions of multi-step transitions that are monitored by the strength of the elastic frustration.

\section{The model Hamiltonian}

The spin-crossover chain is modeled as a homogeneous set of atoms (here sites) which can occupy two states, namely LS and HS, to which we associate a fictitious spin state, $S$, whose eigenvalues are respectively, $S=-1$ and $S=+1$. The lattice sites are coupled by springs and are constrained to move only along the direction of the chain. Each site is coupled elastically to its nearest neighbors (nn) by a spring of elastic constant $A_{0}$ and to its next-nearest-neighbors by another spring of constant elastic, $B_{0}$. The equilibrium distances between two nn sites $i$ and $j$, is noted $R_{e q}^{n n}\left(S_{i}, S_{j}\right)$, and depend on their spin states. Similarly, the equilibrium distance between two nnn sites $i$ and $k$, is noted $R_{e q}^{n n n}\left(S_{i}, S_{k}\right)$. The dependence of the equilibrium distances (i.e. the lattice parameters of the chain) on the spin states is written so as to ensure a bigger length of the chain in the HS state compared to that of the LS state. A schematic view of three electro-elastic configurations (HS-HSHS, HS-LS-HS and LS-LS-LS) of a central site surrounded by its nearest-neighbors is given in Fig. 1. 

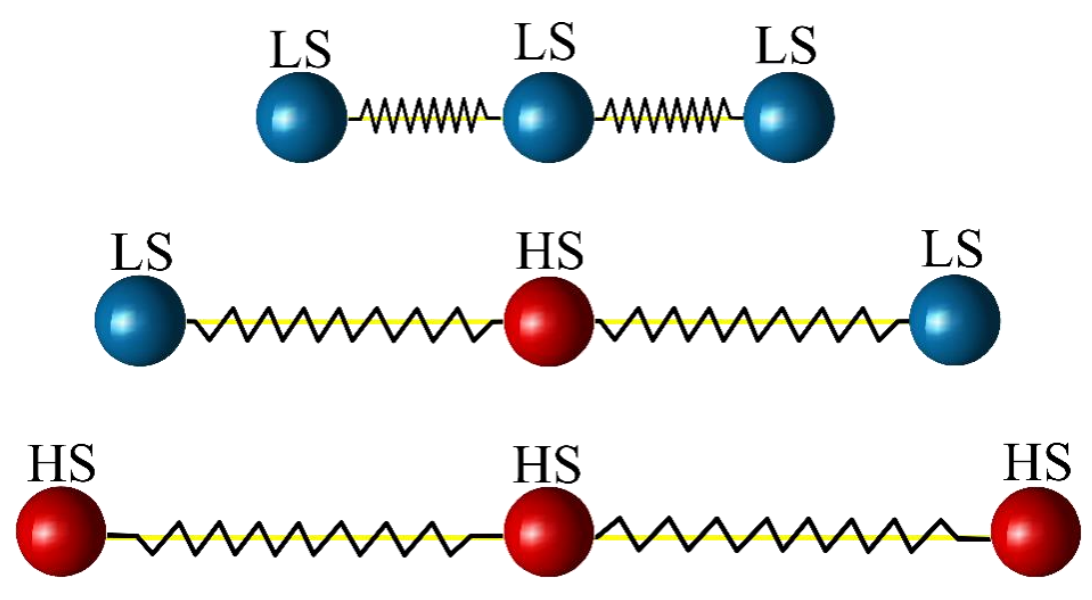

Figure 1: A schematic view of three electronic configurations of the central site and its nearestneighbors. The distance between the sites depends on the spin states. For simplicity, only three configurations among the eight possible states are depicted here.

At variance from the first electronic models, using Ising-like approaches ${ }^{29}$, discrete elastic descriptions based on deformable lattices ${ }^{40-50}$ were introduced so as to mimic the spatiotemporal features revealed by optical microscopy investigations ${ }^{51-53}$ for the nucleation and growth of the spin state phases at the thermal transition of SCO single crystals. The present Hamiltonian of the SCO chain accounting for the electronic and elastic contributions is adapted from our previous electro-elastic model ${ }^{46,54}$ that we solved for several types of crystal shapes and sizes ${ }^{55-61}$. The 1D version of the electro-elastic model writes

$$
H=\sum_{i} \frac{1}{2}\left[\Delta-k_{B} T \ln (g)\right] S_{i}+A_{0} \sum_{i}\left[r_{i, i+1}-R_{0}^{n n}\left(S_{i}, S_{i+1}\right)\right]^{2}+B_{0} \sum_{i}\left[r_{i, i+2}-R_{0}^{n n n}\left(S_{i}, S_{i+2}\right)\right]^{2}
$$

Before to discuss the origin of each term of Hamiltonian (1), it is important to mention here, for the non-specialist reader, that the magnetic exchange between spin-crossover atoms is negligible due to the large distance $(\sim 10 \AA)$ which separates two iron sites. As a matter of fact, except the Prussian blue analogues ${ }^{62-63}$, and with one exception ${ }^{64}$ almost all molecular SCO materials do not show any magnetic ordering at low-temperature, and so the absence of the magnetic exchange between the SCO sites.

The first term of (1) stands for the electronic contribution of the ligand-field energy $\Delta$ at $0 \mathrm{~K}$. The second term, $-k_{B} T \ln g$, is an entropic term originating from the difference of effective degeneracies (electronic and vibrational) between the LS and the HS states, whose ratio, written 
here, $g=\frac{g_{H S}}{g_{L S}}$, is assumed to be temperature-independent. The molar entropy change at the transition, $\Delta S=R \ln g$, easily derived from calorimetric data ${ }^{14}$, which allows the determination of g values, ranging from 100 and 2000. The second and the third terms of (1) describe the elastic interactions between the nearest- (nn) and next-nearest-neighbors (nnn), respectively. Here, the elastic constants $A_{0}$ and $B_{0}$ are assumed, for simplicity, as independent on spin and distortion variables.

Thus in this model, the nn (resp. nnn) $i$ and $i \pm 1$ (resp. $i$ and $i \pm 2$ ) sites of the lattice with corresponding spins $S_{i}$ et $S_{i \pm 1}$ (resp. $S_{i}$ et $S_{i \pm 2}$ ) are linked through springs whose bond-lengths at equilibrium are noted $R_{0}^{n n}\left(S_{i}, S_{j}\right)$ (resp. $R_{0}^{n n n}\left(S_{i}, S_{k}\right)$ ). The atoms are constrained to move only along the $x$-direction. Let's denote by, $R_{0}^{H H}=R_{0}(+1,+1), R_{0}^{L L}=R_{0}(-1,-1)$ et $R_{0}^{H L}=$ $R_{0}(+1,-1)$ the respective nn distances between the sites in the HH, HL and LL. In addition, we consider that $R_{0}^{H L}=\frac{1}{2}\left(R_{0}^{H H}+R_{0}^{L L}\right)$, which means that the equilibrium distance between HS and LS sites is exactly equal to the average distance between those of LS-LS and HS-HS configurations. These electro-elastic configurations are summarized in Fig. 1, where we draw some possible situations. It is quite easy to demonstrate that for the 1D chain, the general expressions of the nn and nnn equilibrium distances connect to the spin states through the simple formulae:

$$
\begin{gathered}
R_{e q}^{n n}\left(S_{i}, S_{j}\right)=R_{0}^{H L}+\frac{\delta_{R}}{4}\left(S_{i}+S_{j}\right) \\
R_{e q, 0}^{n n n}\left(S_{i}, S_{k}\right)=2 R_{0}^{H L}+\frac{1}{2} \delta_{R}\left(S_{i}+S_{k}\right)=2 R_{e q}^{n n}\left(S_{i}, S_{k}\right)
\end{gathered}
$$

Where the quantity $\delta_{R}=\left(R_{0}^{H H}-R_{0}^{L L}\right)$ is the lattice parameter misfit between the LS and HS phases.

Within these equilibrium distances, the full LS and HS states are free from elastic frustration. Indeed, according to Eqs. (2)-(3), when the lattice is fully LS (resp. HS), the nn equilibrium distance, given by Eq. (2), is equal to $R_{e q}^{n n}\left(S_{i}=-1, S_{j}=-1\right)=\frac{1}{2} R_{e q, 0}^{n n n}\left(S_{i}=-1, S_{j}=-1\right)$ [resp. $\left.R_{e q, 0}^{n n}\left(S_{i}=+1, S_{j}=+1\right)=\frac{1}{2} R_{e q}^{n n n}\left(S_{i}=+1, S_{j}=+1\right)\right]$, realizes the constraint of Eq. (3) for the nnn distances. As a result, the total elastic energy is null in both LS and HS states. 
The idea of elastic frustration, at the heart of this paper, relates to the existence of a lattice mismatch between the nn and nnn distances, leading to antagonist equilibrium distances, similarly to Frenkel-Kontorova model ${ }^{65}$, although there are fundamental differences. From the experimental point of view, these behaviors may originate from elastic constraints imposed by the interactions between ligands of adjacent molecules who generally have extended spatial occupations ${ }^{44,21,40}$. Thus, some strong $\pi-\pi$ stacking may contribute to ferro-elastic interactions, while other degrees of freedom (like "weak" hydrogen-hydrogen bonding) may hinder the volume expansion of the molecule from LS to HS. In the present model, and to fully meet the experimental data ${ }^{14}$, we chose to keep the HS state free from any elastic frustration. In contrast, we impose an elastic frustration between the nn and nnn distances for the other configurations, i.e; HS-LS and LS-LS. The elastic frustration is introduced in the nnn equilibrium distance between sites, $i$, and, $k$, as follows (see supplemental material for its derivation),

$$
R_{e q}^{n n n}\left(S_{i}, S_{k}\right)=2 R_{e q}^{n n}\left(S_{i}, S_{k}\right)+\delta_{R} \xi\left(1-\frac{\left(S_{i}+S_{k}\right)}{2}\right)
$$

Where, $\xi$, is a parameter expressing the frustration strength. Using expression (4), the HS state $\left(S_{i}=+1, S_{k}=+1\right)$ is indeed non-frustrated, since $R_{e q}^{n n n}(+1,+1)=2 R_{0}^{H H} \forall \xi$. When $\xi>0$, the equilibrium distances between nnn pairs for LL and HL configurations are respectively

$$
R_{e q}^{n n n}(-1,-1)=2 R_{0}^{L L}+2 \delta_{R} \xi \text { and } R_{e q}^{n n n}( \pm 1, \mp 1)=2 R_{0}^{H L}+\delta_{R} \xi
$$

while those of nn sites are maintained to $R_{0}^{L L}$ and $R_{0}^{H L}$, respectively.

Table 1: Nearest-neighbors and next-nearest neighbors spin configurations of a system of three spins and their associated equilibrium distances showing the antagonist character introduced by the frustration parameter $\xi$. For $\xi=$ $0, \mathrm{nn}$ and nnn fit each other.

\begin{tabular}{|l|c|c|c|}
\hline spin Configurations & \multicolumn{2}{|c|}{ nn equilibrium distances } & nnn equilibrium distance \\
\hline HS-HS-HS & $R_{0}^{H H}$ & $R_{0}^{H H}$ & $2 R_{0}^{H H}$ \\
\hline HS-LS-HS & $R_{0}^{H L}$ & $R_{0}^{L H}$ & $2 R_{0}^{H L}+\delta_{R} \xi$ \\
\hline LS-LS-HS (= HS-LS-LS) & $R_{0}^{L L}$ & $R_{0}^{L H}$ & $2 R_{0}^{H L}+\delta_{R} \xi$ \\
\hline LS-HS-LS & $R_{0}^{L H}$ & $R_{0}^{H L}$ & $2 R_{0}^{L L}+2 \delta_{R} \xi$ \\
\hline HS-HS-LS (=LS-HS-HS) & $R_{0}^{H H}$ & $R_{0}^{H L}$ & $2 R_{0}^{H L}+\delta_{R} \xi$ \\
\hline LS-LS-LS & $R_{0}^{L L}$ & $R_{0}^{L L}$ & $2 R_{0}^{L L}+2 \delta_{R} \xi$ \\
\hline
\end{tabular}


According to Table 1, where we have reported the eight spin configurations of a cluster of three neighboring sites, we see clearly that when the system is fully LS, for example, the elastic frustration parameter $\xi$ introduces antagonist equilibrium distances between nn and nnn distances. Indeed, the $\mathrm{nn}$ distance should lead to a total $\mathrm{nnn}$ equilibrium distance equal to $2 R_{0}^{L L} \quad$ while the $\mathrm{nnn}$ distance imposes an equilibrium distance equal to $2 R_{0}^{L L}+2 \delta_{R} \xi$. The emerging $\mathrm{nn}$ and nnn equilibrium distances will be then the result of the optimization of the elastic energy, and its value will obviously depend on the strengths of the nn and nnn elastic constants, as it will be seen later. To be general, the elastic frustration will always appear as as soon as there is a LS site in the system since the nnn equilibrium distances in LL and HL configurations are bigger than their non-frustrated values, $2 R_{0}^{L L}$ and $2 R_{0}^{H L}$.

In the simulations, we considered the following values for the non-frustrated equilibrium distances, $R_{0}^{L L}=1 \mathrm{~nm}, R_{0}^{H H}=1.2 \mathrm{~nm}, R_{0}^{H L}=1.1 \mathrm{~nm}$, which gives, $\delta_{R}=0.2 \mathrm{~nm}$. The elastic constants have been fixed to, $A_{0}=B_{0}=14000 \mathrm{~K} . \mathrm{nm}^{-2}=14 \mathrm{meV} . A^{-2}$. These values lead to an estimated average bulk modulus, $\mathrm{G} \approx \mathrm{A} \_0 / \mathrm{R} \_0 \sim 8 \mathrm{GPa}$, which is in excellent agreement with available experimental data of Brillouin scattering performed on the single crystal of $[\mathrm{Fe}(\mathrm{ptz}) 6](\mathrm{ClO} 4) 2$ which led to bulk modulus values found in the range 5-20 GPa ${ }^{66-67}$. The value of the ligand-field energy is $\Delta_{0}=450 \mathrm{~K}$ and that of the degeneracy ratio, $\ln g=5$. This value leads to an entropy change at the transition $\Delta S=41 \mathrm{~J} . \mathrm{K} \cdot \mathrm{mol}^{-1}$ in good agreement with experimental data of heat capacity measurements ${ }^{14}$.

\section{Results and discussion:}

The spin transition is described here through the usual variables, among which the HS fraction, $\mathrm{n}_{H S}$, which connects to the average magnetization $\langle S\rangle$, and also through fraction $n_{H L}$, of neighboring HS-LS pairs, as well as through the average value of the lattice parameter $\langle d\rangle$. These quantities can be expressed simply as,

$$
n_{H S}=\frac{(1+<S>)}{2}, n_{H L}=\frac{1-<S_{i} S_{j}>}{4} \text { and }\left\langle d>=\frac{\sum_{i j}\left(x_{j}-x_{i}\right)}{(N-1)}\right.
$$


Where, $\langle\mathrm{S}\rangle$ is the average spin value, $\left\langle S_{i} S_{j}\right\rangle$ is the average value of nn spin-spin correlation, and $\left(x_{j}-x_{i}\right)$ is the distance between neighboring sites $i$ and $j$.

In view of the complex structure of Hamiltonian (1) in which spin and distortion variables are intricate, and so which cannot be solved analytically, we use the Monte Carlo (MC) technique to study its thermal properties considering the frustration strength $\xi$ as a control parameter.

The simulations are performed on a chain of 60 sites and the MC simulations concerned both spin and lattice position variables, using a sequential procedure. The stochastic algorithm is performed in the following way: for a site $i$ randomly selected, with spin $S_{i}= \pm 1$ and position, $x_{i}$, a new spin value $S_{i}^{\prime}=-S_{i}$ is set without position change. This spin change is accepted or rejected by the usual Metropolis criterion. Once the new spin value is accepted then the lattice is relaxed mechanically by a slight motion of nodes (selected randomly) with a quantity $\delta x=0.001$ which is much smaller than the distance between the spin states. The procedure of the lattice relaxation is repeated 10 times for each spin flip. Afterwards, a new site will be selected randomly and so on ... Once all nodes of the lattice are visited for the spin change, we define such step as the unit of the Monte Carlo step and denoted "MCS". In the present simulations, the thermal properties are calculated by changing the temperature each by $1 \mathrm{~K}$ step. At each temperature, we perform $10000 \mathrm{MCS}$ to reach the equilibrium state and we use 1000 other MCS for the statistics. Within this procedure, each site is displaced 600 times for $1 \mathrm{MCS}$. So, at each temperature, each spin state and lattice position are updated $10^{7}$ and $610^{9}$ times, respectively. We have checked that increasing the simulation time does not affect the final results, which ensures that we reached the stationary state for spin and lattice position variables.

Figs. 2a and 2b summarize the system's behavior for two values of the elastic constants, $A_{0}=$

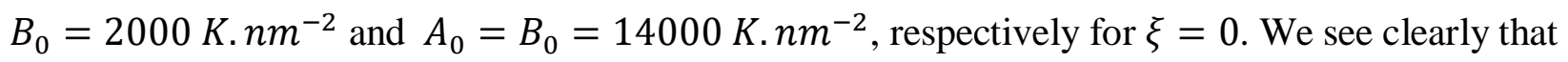
while the chain presents a gradual transition between the LS and HS states for weak elastic constants, it clearly undergoes a "first-order transition" with a large thermal hysteresis, a characteristic of a strong cooperative SCO system, for the case of a strong elastic interaction. A selected number of snapshots showing the spatial distribution of spin states at different temperatures along the spin transitions are presented in Fig. S1 of the supplemental material. In both cases, the change of the length of the chain at the transition is well identified. 
Let us now discuss the presence of the hysteretic behavior of Fig. 2b, even though that the system is $1 \mathrm{D}$. This thermal hysteresis has a kinetic origin and can be attributed to finite size effects. Indeed, as we have demonstrated in a previous work ${ }^{33}$ devoted to the study of dynamic phase transitions in 1D spin-phonon coupling model, which constitutes a variant of the present Hamiltonian, the kinetic hysteresis widths increases almost linearly with the inverse of the chain length. That being said, it should also be highlighted that since the two simulations are performed using the same temperature scan rate, the presence of the kinetic hysteresis in Fig. $2 b$ is clearly a strengthened by the increase of the elastic cooperativity inside the system.

The strength of the cooperative interactions is identified here as proportional to the misfit of elastic energy per site, $\left(A_{0}+2 B_{0}\right) \delta_{R}^{2}$. It is worth noticing here, that this behavior contrasts with that of the usual Ising $1 \mathrm{D}$ chains with short-range interactions, the kinetic hysteresis of which are less wider. One may think that the presence of elastic interactions, which are known to lead to longrange interactions, making the system belonging to mean-field universality class ${ }^{68}$ at least for a two-dimensional system, clearly lead to larger energy barriers which may then enhance the existence of these kinetic thermal hysteresis. In the experimental systems 1D spin-crossover systems, the SCO chains are rarely isolated and several types of interactions may connect them, starting with weak hydrogen bonding to strong stacking, which then leads to 3D structures which however should keep the memory of their strong 1D character. In such situations, true first-order transitions or very sharp transitions are allowed.

At the transition temperature, $T_{1 / 2}^{0}$, we fulfill the relation $\left\langle S>=0\left(n_{H S}=0.5\right)\right.$ and $<d>=$ $R_{0}^{H L}$. According to the general law that at the transition, the total effective ligand field becomes equal to zero, we simply obtain from Hamiltonian (1),

$$
T_{1 / 2}^{0}=\frac{\Delta}{k_{B} \ln (g)}
$$


(a)

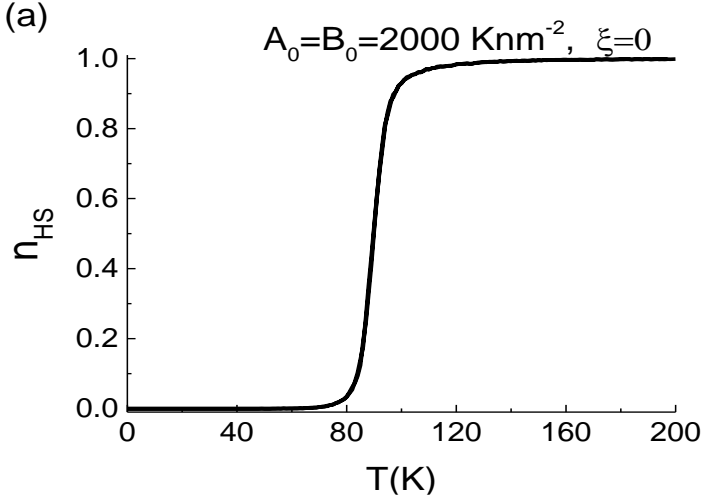

(b)

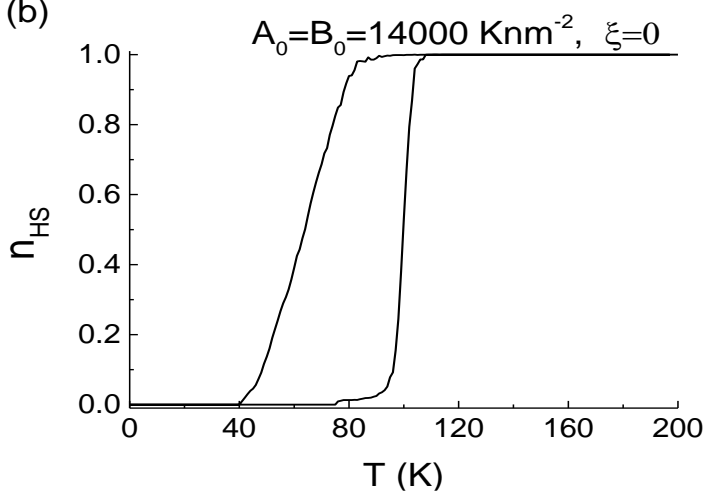

Figure 2: a) Thermal dependence of the HS fraction $n_{H S}$ in the non-frustrated case $(\xi=0)$ for two different set of values of the elastic interactions: (a) the case of weak coupling, $A_{0}=B_{0}=2000 \mathrm{~K} . \mathrm{nm}^{-2}$ leading to a gradual spin transition, and $\mathrm{b}$ ) the case of strong coupling $A_{0}=B_{0}=14000 \mathrm{~K} \cdot \mathrm{nm}^{-2}$ leading with the same temperature scan rate to a hysteretic behavior. The thermal hysteresis has a kinetic origin. (see text for more explanation).

(a)

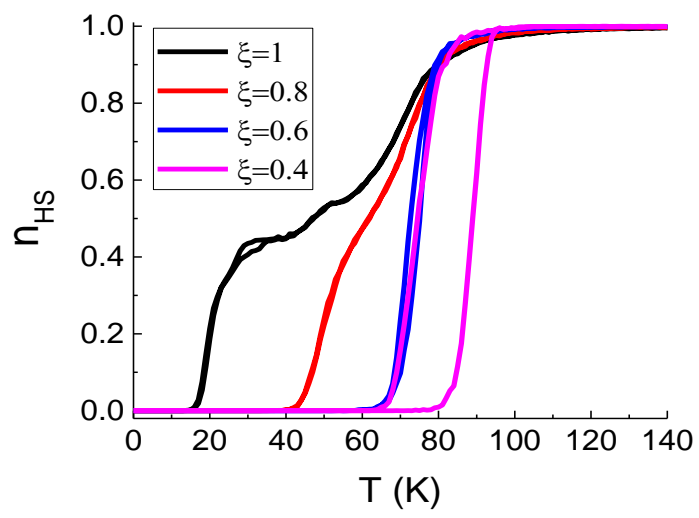

(b)

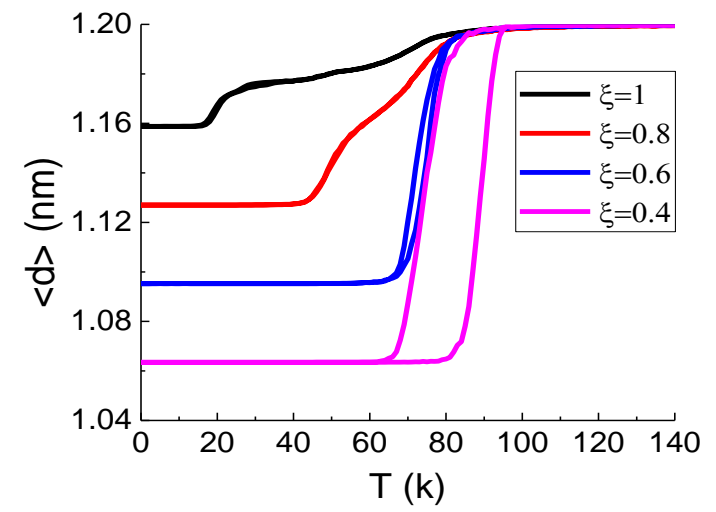

(c)

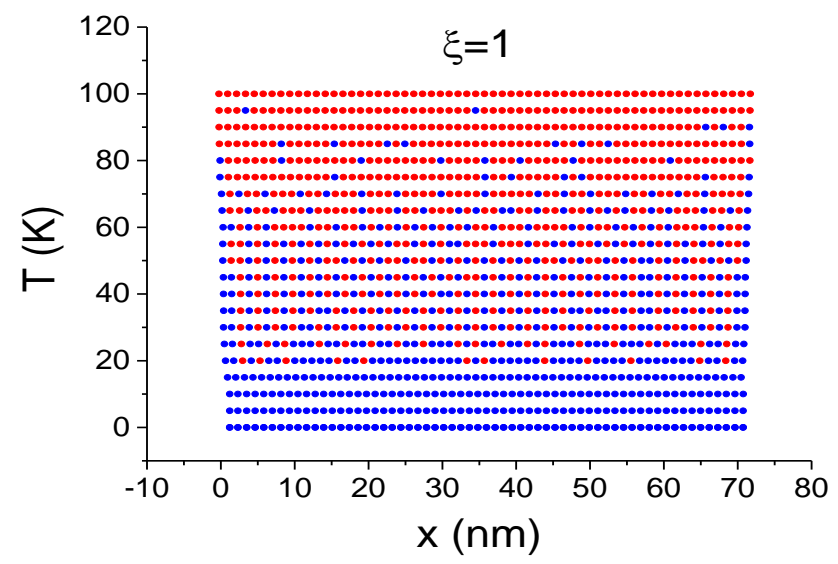

Figure 3: a) MC results of the thermal dependence of the HS fraction $n_{H S}$ of Hamiltonian (1) for different values of the elastic frustration strength, $\xi$, showing the first-order transition with hysteresis (pink and blue), as well two-step 
transitions with a plateau (red and black). b) Corresponding thermal dependence of the average nn distances. Notice the monotonous increase of the relaxed lattice parameter of the LS state (i.e. $\left\langle r_{i j}\right\rangle$ at $0 \mathrm{~K}$ ) as $\xi$ increases. c) spatial distribution of the spin states (red=HS and blue=LS) along the thermal transition for $\xi=1$, showing a selforganization of the spin states in the plateau region. The parameter values are $R_{0}^{L L}=1 \mathrm{~nm}, R_{0}^{H H}=1.2 \mathrm{~nm}, R_{0}^{H L}=$ $1.1 \mathrm{~nm}, \delta_{R}=0.2 \mathrm{~nm}, A_{0}=B_{0}=14000 \mathrm{~K} . \mathrm{nm}^{-2}=14 \mathrm{meV} . A^{-2}, \Delta_{0}=450 \mathrm{~K}, \ln \mathrm{g}=5$. Here too, the hysteresis appearing in a) and b) have a kinetic origin.

When the elastic frustration between $\mathrm{nn}$ and nnn sites comes into play in the strong cooperative case, the curve of the HS fraction is moved to low-temperature regions and the spin transition transforms from first-order with hysteresis to a two-step transition with a large plateau around $n_{H S}=0.5$ (red and black cures of Fig. 3a). This behavior appears beyond some threshold value of, $\xi$. Thus, for small values of the frustration parameter, $\xi$, one obtains again the usual "firstorder transition" between LS and HS states, but the width of the hysteresis is severely reduced. Increasing $\xi$ values, results in a change of the thermal dependence of the HS fraction into a twostep spin transition, Figure $3 \mathrm{~b}$ allows stablishing a close relation between the elastic and electronic properties of the lattice. As a matter of fact, the low-temperature value of the average lattice parameter, $\langle d\rangle$, increases monotonously with $\xi$ and when its value exceeds $R_{0}^{H L}=1.1 \mathrm{~nm}$ (see red curve in Fig. 3b), a plateau starts to emerge in the thermal dependence of $n_{H S}$ and $\langle d\rangle$. To get a clearer idea of the organization mode of the spin states inside the plateau region, we plotted in Fig. 3c, the spatial configurations of the spin states (red dots=HS and blue dots=LS) inside the lattice along the thermal transition of the two-step behavior obtained for $\xi=1$ in Fig. 3a. A clear evidence of the presence of an ordered state HS-LS-HS-LS, noted for simplicity HL, is observed in the plateau region, i.e. for $40 \leq T \leq 45 \mathrm{~K}$. This behavior is confirmed by the study of the temperature-dependence of the proportion of neighboring HS-LS pairs (obtained from MC simulations), $n_{H L}(T)$, which is presented in Fig. S2 of the SM. One expects this quantity to increase around the transition temperatures and to fall down in the LS and HS states. This is indeed the case for $\xi=0.4$ and 0.6 , where small peaks are visible on their associated curves. The amplitude of the maximum increases as $\xi$ increases, and reaches the value, $n_{H L} \sim 0.5$ for $\xi=1$ in the temperature interval $25-60 \mathrm{~K}$. Since, $n_{H L}=n_{L H}$, it clearly appears that the presence of this large maximum is the signature of the presence of an ordered state HS-LS-HS-LS, which will be noted for simplicity as (HLHLHL...) 
To confirm these facts, we display in Figures $4 a$ and $4 b$ the spatial organization of the spin states in the plateau region of the black curve of figure 3 corresponding to the value, $\xi=1$ at a temperature $T=40 \mathrm{~K}$, situated in the middle of the plateau region. An antiferromagnetic-like (HLHLHL...) order inside the plateau, where each HS (resp. LS) molecule is surrounded by nn LS (resp. HS) molecules is then confirmed. In addition, one can see the presence antiphase boundaries, highlighted by the green squares, because the symmetric configuration (LHLHLH...) has the same energy.
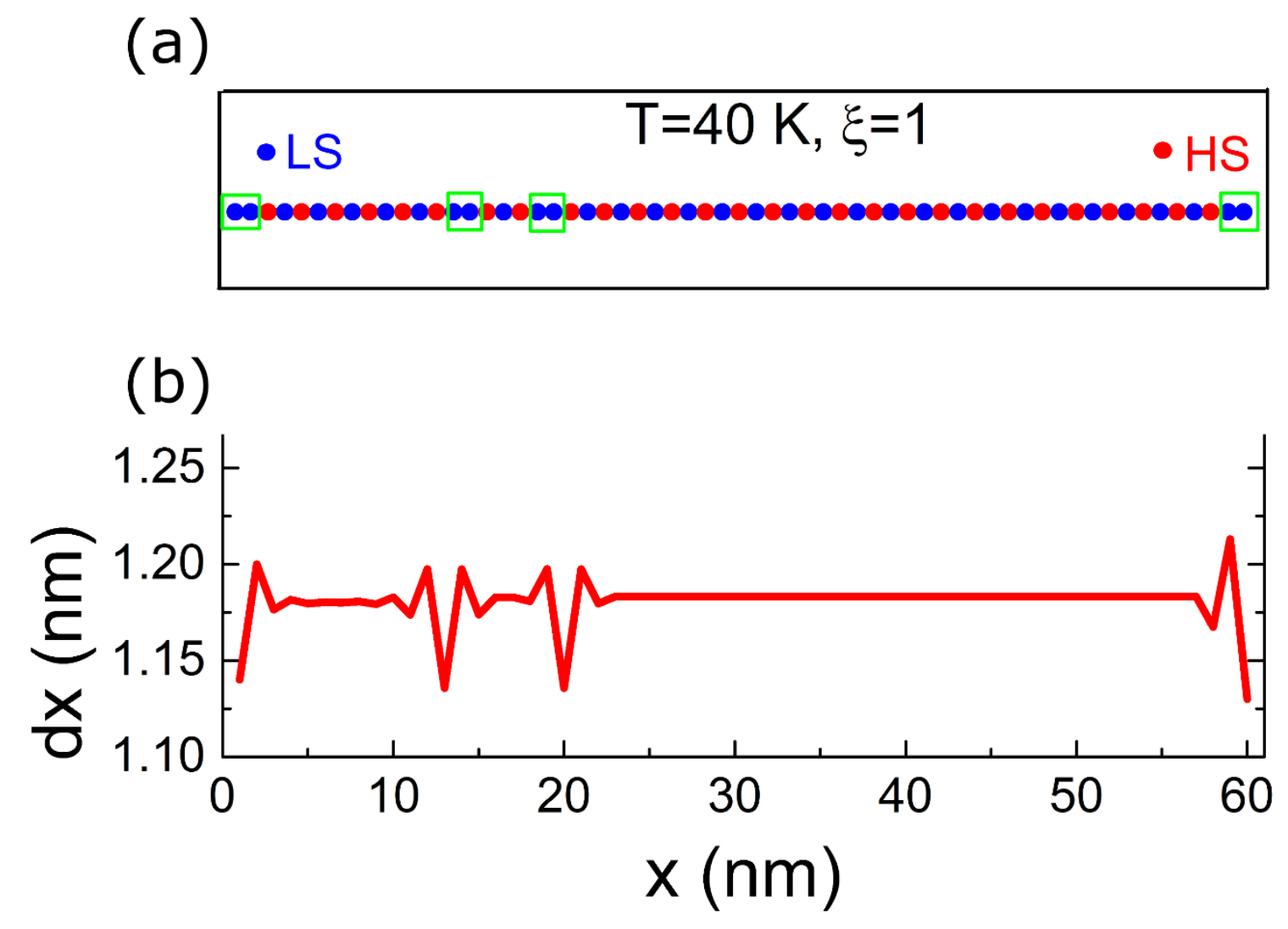

Figure 4: a) Snapshot of the spatial distribution of the spin states of a chain of 60 sites, showing an antiferromagnetic-like organization in the plateau region of the black curve of Fig. 1a at $T=40 \mathrm{~K}$ and $\xi=1$. b) Space-dependence of the distance between nn successive atoms along the chain, showing a constant value except at the border of the chain and around the anti-phase boundaries LL where $d_{x}$ falls down. An excellent correlation is observed between the electronic and the structural behaviors. 
To clarify the role of the elastic interactions in the emergence of the antiferromagnetic-like ordering, we draw the space dependence of the nn inter-site distances along the chain. The results, presented in Fig. 4b, shows that the interatomic distance, $d_{x}=x_{i+1}-x_{i}$, keeps a constant value, $d_{x}=1.18 \mathrm{~nm}$, in the antiferromagnetic-like region, and shows some local variation around the antiphase boundaries. Interestingly, and as expected, in the region of a monotonous lattice parameter, a region with consecutive HS-LS configurations, the average distance $d_{x}$ is different from the nn value, $R_{0}^{H L}=1.1 \mathrm{~nm}$. Due to the ordered structure of the system, only two kinds of nnn configurations are found: namely HS-LS-HS or LS-HS-LS. The latter imposes nnn equilibrium distances equal to $2 R_{0}^{H H}=2.4 \mathrm{~nm}$ for the first case, and $2 R_{0}^{L L}+2 \delta_{R} \xi=2.4 \mathrm{~nm}$ (see Table 1 ) for the second case. The interatomic distance $d_{x}=1.18 \mathrm{~nm}$ of the mechanically relaxed state emerging from the simulations is then the distance which minimizes the total elastic energy, and clearly, its dependence on the elastic constants is non-linear. So, to consider this important problem from a general view point, we propose in the following a simple analytical procedure, to determine the expressions of these relaxed nn distances.

\section{III.1 Analytical expressions of the relaxed lattice parameters:}

To understand the behavior of the elastic properties of the model, which monitor those of the HS fraction, we study analytically the $\xi$-dependence of the relaxed nn distance, $d_{x}$, in three selected ordered electronic configurations of interest, of Fig. 3, namely HH (=HS-HS-HS...), LL (=LS-LSLS...) and HL (=HS-LS-HS-LS...), corresponding respectively to a system fully HS, fully LS and antiferro-magnetically ordered. The thermal dependence of the average nn distance,

First, when the lattice adopts the HS phase, there is no elastic frustration, and so its minimum elastic energy is always zero, $\forall \xi$, that is because the equilibrium nn and nnn distances are $R_{0}^{H H}$ and $2 R_{0}^{H H}$, respectively. In contrast, the LS and the ordered antiferro-like structure are frustrated for $\xi \neq 0$, and their total relaxed elastic energies remain non-zero, due to the incompatibility between their nn and nnn equilibrium distances, as previously discussed.

Let us denote by $R_{L L}^{\text {relax }}$ and $R_{H L}^{\text {relax }}$ the relaxed nn distances corresponding to LL and HL macroscopic states. We assume that the lattice parameter is homogeneous along the chain. Let's denote by $x$ (resp. $2 x$ ) the distance between the nn (resp. nnn), then it follows from Hamiltonian 
(1) that the total non-relaxed energy of the system (including electronic and elastic contributions) in these configurations write as

$$
\begin{aligned}
& E_{H H}=N \Delta_{e f f}+(N-1) A_{0}\left(x-R_{e q}^{n n}(+1,+1)\right)^{2}+(N-2) B_{0}\left(2 x-R_{e q}^{n n n}(+1,+1)\right)^{2} \\
& E_{L L}=-N \Delta_{e f f}+(N-1) A_{0}\left(x-R_{e q}^{n n}(-1,-1)\right)^{2}+(N-2) B_{0}\left(2 x-R_{e q}^{n n n}(-1,-1)\right)^{2} \\
& E_{H L}=(N-1) A_{0}\left(x-R_{e q}^{n n}(-1,+1)\right)^{2}+\frac{(N-2)}{2} B_{0}\left(2 x-R_{e q}^{n n n}(+1,+1)\right)^{2}+\frac{(N-2)}{2} B_{0}(2 x- \\
& \left.R_{e q}^{n n n}(-1,-1)\right)^{2}(8 \mathrm{c})
\end{aligned}
$$

Substituting $R_{e q}^{n n}\left(S_{i}, S_{j}\right)$ and $R_{e q}^{n n n}\left(S_{i}, S_{k}\right)$ by their expressions given in Eqs. (2) and (4), the elastic energy densities in the case of a long chain $(N \gg 1)$ become :

$$
\begin{gathered}
E_{L L}=-\Delta_{e f f}+A_{0}\left(x-R_{0}^{L L}\right)^{2}+4 B_{0}\left(x-R_{0}^{L L}-\delta_{R} \xi\right)^{2} \\
E_{H L}=A_{0}\left(x-R_{0}^{H L}\right)^{2}+2 B_{0}\left(x-R_{0}^{H H}\right)^{2}+2 B_{0}\left(x-R_{0}^{L l}-\delta_{R} \xi\right)^{2} \\
E_{H H}=\Delta_{e f f}+\left(A_{0}+4 B_{0}\right)\left(x-R_{0}^{H H}\right)^{2} . \quad(9 \mathrm{c})
\end{gathered}
$$

The relaxed distances are obtained by minimizing these expressions with respect to $x$, which leads to,

$$
\begin{gathered}
x_{H H}^{\text {relax }}=R_{0}^{H H} \\
x_{L L}^{\text {relax }}=R_{0}^{L L}+\frac{4 \xi}{k+4} \delta_{R} \\
x_{H L}^{\text {relax }}=R_{0}^{H L}+\frac{2 \xi}{k+4} \delta_{R}
\end{gathered}
$$

Where $k=\frac{A_{0}}{B_{0}}$ is the ratio between the nn and nnn elastic constants. The linear $\xi$-dependence of the three relaxed nn distances in the LL, HL and HH states is represented in Fig. S3 of the supplemental material, where the reader can remark the existence of a particular point $\xi_{c}=1.25$ above which the relaxed HL and LL distances become bigger than that of the HS state.

Eqs. (10b-c) bring to light, the non-linear dependence of the relaxed nn distance with the elastic constants. Moreover, we see that the relaxed nn distances in the LS $\left(x_{L L}^{\text {relax }}\right)$ and in the ordered antiferro-like HS-LS state, $\left(x_{H L}^{\text {relax }}\right)$, are bigger than their respective non-frustrated values, $R_{0}^{L L}$ and $R_{0}^{H L}$ they are simply linear increasing functions of the frustration parameter $\xi>0$ and the lattice 
parameter misfit $\delta_{R}$. In the case where, $B_{0} \gg A_{0}$, the nn potential dominates which leads to $k \sim 0$, and the frustration becomes maximum.

To check the reliability of these analytical predictions, we compared in Fig. 5 the analytical values of, $R_{L L}^{\text {relax }}(\xi)$, in the LS state, given by Eq. (10b), with those derived from the MC simulations of Fig. 4, at $0 \mathrm{~K}$. An excellent agreement is found between these two sets of data, thus confirming the relevance of the present approach.

According to Eqs. (10), the elastic frustration, tends to expand locally the lattice parameter in the LL and HL states, resulting in the stabilization of the HS state. This fact is identified as the main reason for which the representative curves of the HS fraction $n_{H S}(T)$ (Fig. 2) shift to lower temperature region when increasing $\xi$ values.

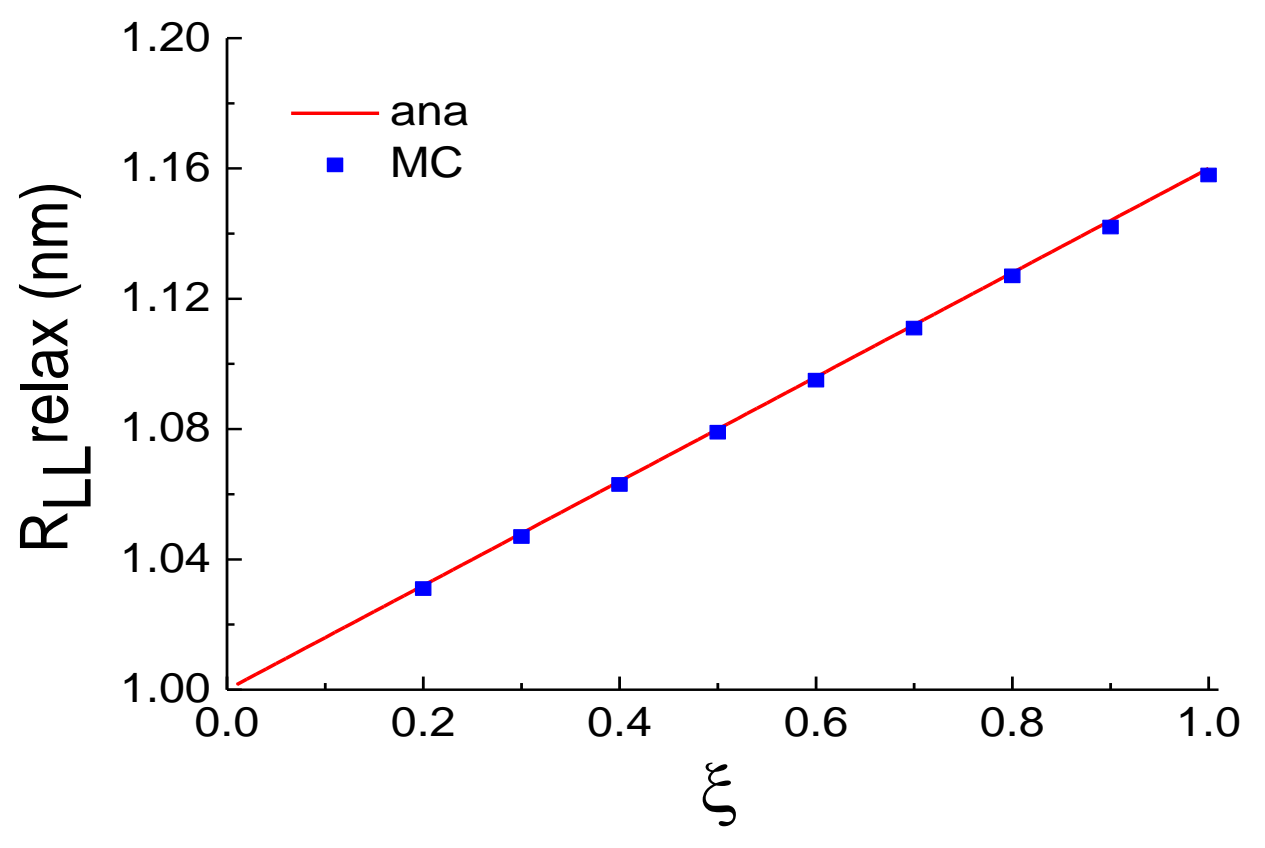

Figure 5: Relaxed nn distance, $R_{L L}^{\text {relax }}$, in the LS state vs elastic frustration strength, $\xi$. The blue squares are MC data and the red line corresponds to the analytical prediction of Eq. (10b). The calculations are performed at $T=10 \mathrm{~K}$. Remark the excellent quantitative predictions of the analytical approach.

\section{III.2 Frustration-dependence of the total energies}

To go further in the analysis of the thermal dependence of the HS fraction of Fig. 3, we calculate the total relaxed energies in the HH, HL and LL states, which are easily obtained by replacing in 
Eqs. 9a-c, the relaxed nn distances, $R_{H H}^{\text {relax }}, R_{L L}^{\text {relax }}, R_{H L}^{\text {relax }}$ by their expressions in (10a-c). The mechanically relaxed in the ordered LL, HL and HH configurations write,

$$
\begin{aligned}
& E_{L L}^{\text {relax }}=-N \Delta_{e f f}+N\left(\frac{\xi}{\xi_{c}} \delta_{R}\right)^{2}\left[A_{0}+4 B_{0}\left(1-\xi_{c}\right)^{2}\right]=-N \Delta_{e f f}+4 N A_{0} \delta_{R}^{2} \xi^{2} \frac{k^{2}+4}{(k+4)^{2}} \\
& E_{H L}^{\text {relax }}=N\left(\frac{\xi}{2 \xi_{c}} \delta_{R}\right)^{2}\left[A_{0}+2 B_{0}\left(1-\xi_{c}\right)^{2}\right]+\frac{N B_{0}}{2} \delta_{R}^{2}\left(1+\xi\left(\frac{1}{\xi_{c}}-2\right)\right)^{2}=\frac{N B_{0}}{2} \delta_{R}^{2}\left[4 \frac{k+1}{k+4} \xi^{2}-\right. \\
& 4 \xi+1](11 \mathrm{~b})
\end{aligned}
$$

$$
E_{H H}=N \Delta_{e f f}
$$

Where, $\xi_{c}=\frac{k+4}{4}$.

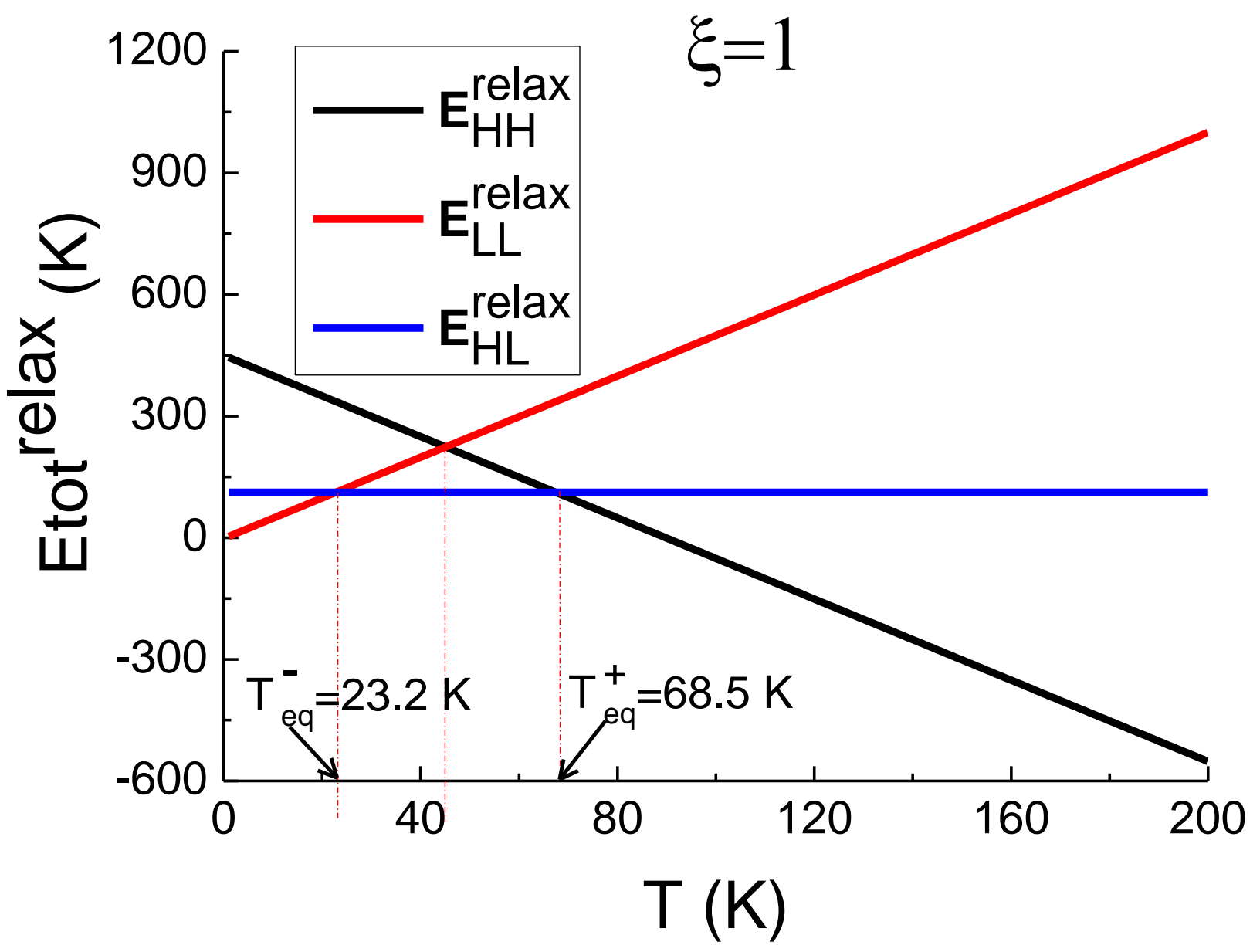


Figure 6: Thermal-dependence of the total analytical energies given by Eqs. (15)-(17) of the three lattice configurations, $\mathrm{LL}, \mathrm{HH}, \mathrm{HL}$, for $\xi=1$. The thermal dependence of the system follows the energy minimum landscape, which leads to A-B-C-D pathway, along which in the plateau B-C (blue curve), the HL state is stabilized. The transition temperatures, $23.2 \mathrm{~K}$ and $68.5 \mathrm{~K}$ at which these intersections take place, are in excellent agreement with MC data of Fig. 3.

The thermal evolution of the total relaxed energies of the LL, HL and HH lattices, depicted in Fig. 6, for the case, $\xi=1$, leads to interesting information. The three linear plots, clearly demonstrate that on both cooling and heating processes, the energy of the LL (in red) and HH (in black) cross that of the HL (in blue) at first. Since the system follows the minimum energy pathway (the states are considered here as ordered and so their entropies are zero), it is found that the antiferromagnetic-like HL state is stabilized in the course of the thermal process, illustrated by the letters A, B, C, D in Fig. 6. Moreover, the numerical values of the predicted transition temperatures, $T^{-}=23.2 \mathrm{~K}$ and $T^{+}=68.5 \mathrm{~K}$, corresponding to $\mathrm{LL} \rightarrow \mathrm{HL}$ and $\mathrm{HL} \rightarrow \mathrm{HH}$ transformations, are in excellent agreement with MC data of Fig. 3. The behavior of the plateau width as a function of $\xi$ is considered in detail in the forthcoming sections.

\section{III.3 Effect of the elastic frustration on the transition temperatures and phase diagram}

At equilibrium thermodynamics, the transition temperature between two coexisting phases correspond to the temperature for which their corresponding free energies are equal, that is the Maxwell point. Here, we aim to find the analytical expressions of the equilibrium temperatures corresponding to the transitions between LL, HL and HH states. For simplicity, we consider that the transition occurs between ordered states, then their associated entropies are equal to zero. Thus, the condition of equal free energies transforms to equal internal energies.

However, one should mention that, in the presence of a first-order transition with a thermal hysteresis, this analytical method will note give the limiting temperatures $\left(T^{-}, T^{+}\right)$of the hysteresis (which depend on the lifetime of metastable states), but will lead to the so-called equilibrium temperatures, while the MC simulations do exactly the contrary. So to compare the results, the equilibrium temperature is then derived from $\mathrm{MC}$ data as $\sim\left(T^{-}+T^{+}\right) / 2$. 
First, we start by determining the transition temperature, $T_{e q}$, corresponding to the hysteresis loops of Fig. 2a, corresponding to cooperative transformation between full LS and HS states. Since the transition takes place between two ordered states, namely LS and HS, their entropies are null, which transforms the condition of equal free energies to that of equal energies, thus giving, $E\left(T=T_{\text {eq }},<S_{i}>=+1, x=R_{\text {relax }}^{H H}\right)=E\left(T=T_{\text {eq }},<S_{i}>=-1, x=R_{\text {relax }}^{L L}\right) . \quad$ Using the computed total energies whose expressions are given in Eqs. (7), one easily arrives at the frustration-dependence of the transition temperature,

$$
T_{e q}(\xi)=\frac{\Delta_{0}}{k_{B} \ln g}-\frac{A_{0} \delta_{R}^{2} \xi^{2}}{2 \ln g}\left[\frac{5}{\xi_{\max }^{2}}-\frac{8}{\xi_{\max }}+4\right],
$$

in which we recognise as first term the transition temperature of the usual Ising-like model. The second contribution is that of the elastic frustration which brings an additional effective ligand field energy to $\Delta_{0}$. As a consequence, the global transition temperature of the system shows a quadratic decrease with the frustration parameter.

The analytical curve $T_{e q}(\xi)$ is plotted in the region I of the phase diagram of Fig. 7, where it excellently agrees with the results of MC simulations.

\section{III.4 The condition for the appearance of the two-step spin transitions:}

The thermal dependence of the HS fraction of Fig. 3a indicates that high values of $\xi$ lead to the emergence of a plateau around $n_{H S} \sim 0.5$ in the thermal transition, where the spin states show an antiferromagnetic-like self-organization (HLHLHL...), denoted here HL for simplicity. Our objective in this section is to find the analytical expressions of the transition temperatures, $T_{e q}^{-}$and $T_{e q}^{+}$, at which occur the LL↔HL and HL↔HS conversions, respectively. For that, one should remark that at these temperatures, the total energies of the elastically relaxed LL, HL and HH states must obey the following conditions

$$
\begin{aligned}
& E\left(T=T_{e q}^{+},<S_{i}>=+1, x=R_{\text {relax }}^{H H}\right)=E\left(T=T_{e q}^{+},<S_{i}>=0, x=R_{\text {relax }}^{H L}\right) \\
& E\left(T=T_{\text {eq }}^{-},<S_{i}>=-1, x=R_{\text {relax }}^{L L}\right)=E\left(T=T_{\text {eq }}^{-},<S_{i}>=0, x=R_{\text {relax }}^{H L}\right)
\end{aligned}
$$


Using the expressions of energies found in Eqs. (11), we arrive immediately after from simplifications to,

$$
T^{+}(\xi)=T_{e q}^{0}-\frac{2 A_{0} \delta_{R}^{2}}{k_{B} \ln g}\left[\frac{5}{8 \xi_{\max }^{2}}-\frac{1}{\xi_{\max }}+1\right] \xi^{2}+\frac{2 A_{0} \delta_{R}^{2}}{k_{B} \ln g}(\xi-1 / 2)
$$

and

$$
T^{-}(\xi)=T_{e q}^{0}+\frac{2 A_{0} \delta_{R}^{2}}{k_{B} \ln g}\left[\frac{-15}{4 \xi_{\max }^{2}}+\frac{6}{\xi_{\max }}-2\right] \xi^{2}-\frac{2 A_{0} \delta_{R}^{2}}{k_{B} \ln g}(\xi-1 / 2)
$$

The two-step transitions exist as soon as $T^{+}(\xi)>T^{-}(\xi)$ and $T^{-}(\xi)>0$. These two conditions impose an interval of $\xi$ values limited here to $0.65<\xi<1.25$ for the present used model parameter values. This behavior corresponds to region 2 of the phase diagram of Fig. 7, where the representative curves of $T^{ \pm}(\xi)$ are displayed and compared to MC data, with which they also show an excellent agreement. Moreover, Eqs. (10) allows to deduce the width of the plateau region as

$$
\Delta T=T^{+}(\xi)-T^{-}(\xi)=\frac{A_{0} \delta_{R}^{2}}{k_{B} \ln g}\left[\frac{10}{4 \xi_{\max }^{2}}-\frac{4}{\xi_{\max }}\right] \xi^{2}+\frac{4 A_{0} \delta_{R}^{2}}{k_{B} \ln g} \xi-\frac{2 A_{0} \delta_{R}^{2}}{k_{B} \ln g},
$$

showing a parabolic increase with the elastic frustration strength.

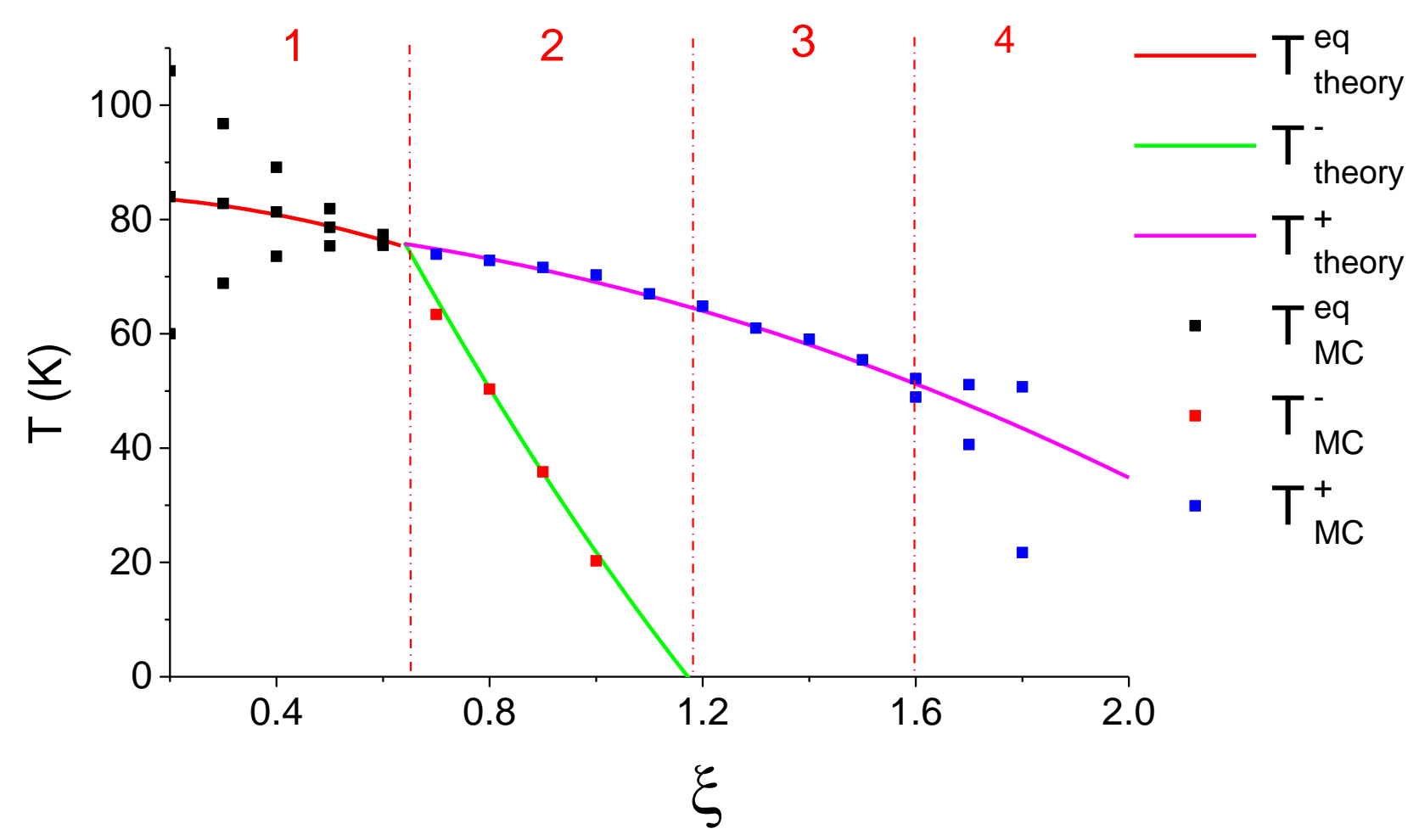


Figure 7: Phase diagram $T(\xi)$ summarizing the behaviour of the transition temperatures vs the frustration parameter $\xi$ obtained by MC (squares) simulations and analytically (continuous lines). Three regions are found: (1) for $0.2<\xi<0.65$, a one-step spin transition between HS and LS with hysteresis. Black squares are $\mathrm{MC}$ data and the red line is the analytical prediction for $T_{e q}(\xi)$. (ii) $0.65<\xi<1.2$ complete two-step transitions region. Red (resp. green) and blue rep. violet) squares (resp. lines) are MC (resp. analytical) data of $T^{-}(\xi)$ and $T^{+}(\xi)$. Region (3), $1.2<\xi<1.65$ is the region of incomplete, two-step but gradual transitions. For $\xi>1.65$ the thermal hysteresis re-appears, announcing the existence of a re-entrance phenomenon.

\section{III.5. Incomplete spin transitions and modulated structures}

A spin transition is usually called incomplete ${ }^{24,40}$, when the conversion on cooling from the full HS state leads at low-temperature to a mixed phase of LS and HS states. This means that there is a residual fraction of HS species which do not convert at very low-temperature. This phenomenon is often observed in spin transition materials and was not considered seriously from the physics side, probably because everybody is looking for complete and hysteretic transitions. However, the existence of a reluctant HS fraction at low-temperature presents challenging problems of elasticity, and one has to understand what is the physical origin behind this very common observation. In the present study, the 1D elastic chains lead to incomplete spin transitions beyond the threshold frustration rate value $\xi \sim 1.2$, as shown in Fig. 8a, where we notice that the system stabilizes at 0 K only a quantified number of residual HS fractions: $n_{H S}=1 / 3, n_{H S}=2 / 3$ and $n_{H S}=3 / 4$ for high $\xi$ values. Concomitantly, the corresponding evolution of the average nn distance at $0 \mathrm{~K}$, shows a continuous and linear increase with respect to $\xi$. Theoretically speaking, the existence of a residual HS fraction at $0 \mathrm{~K}$ requires fulfilling the constraint $R_{L L}^{\text {relax }} \geq R_{0}^{H H}$, which according to Eq. (10b), is expected for $\xi_{c}=\frac{A_{0}+4 B_{0}}{4 B_{0}}$, evaluated to $\xi_{m}=1.25$. A meticulous inspection of the MC data of Fig. 8b giving the thermal-dependence of the average nn distance for several $\xi$ values, shows that the incomplete transition appears sooner in the simulation, i.e. from $\xi=1.2$. The small discrepancy between the analytical predictions and the simulations is attributed to kinetic effects of the MC procedure at low-temperature, which considerably slows down the thermal activation which increases the lifetime of the relaxation processes. These results are summarized in region 3 of the phase diagram of Fig. 7 which displays the $T-\xi$ behavior of incomplete and multi-step transitions. 


\section{(a)}

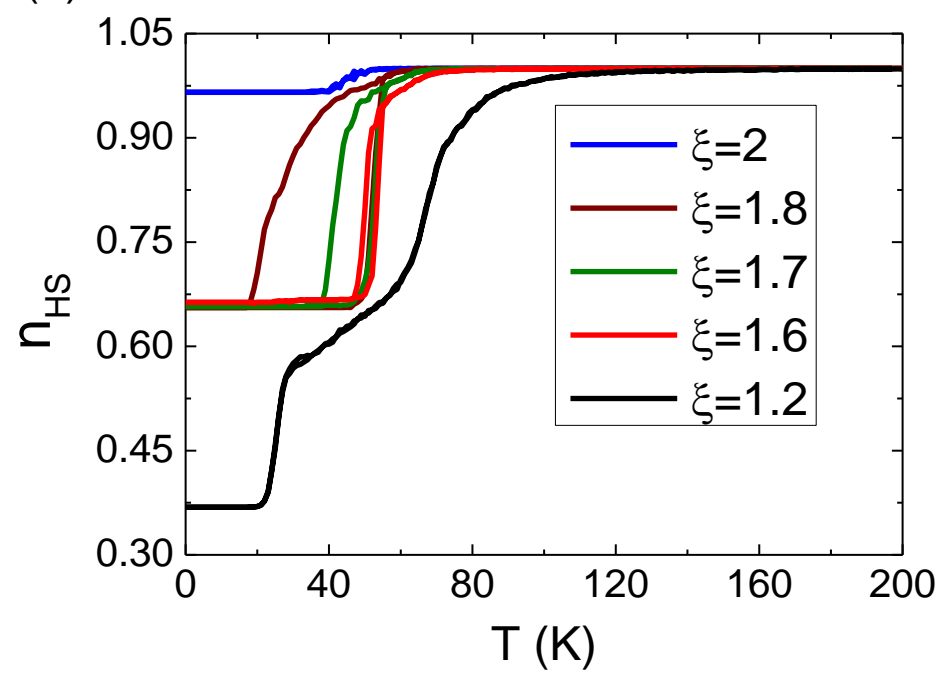

(b)

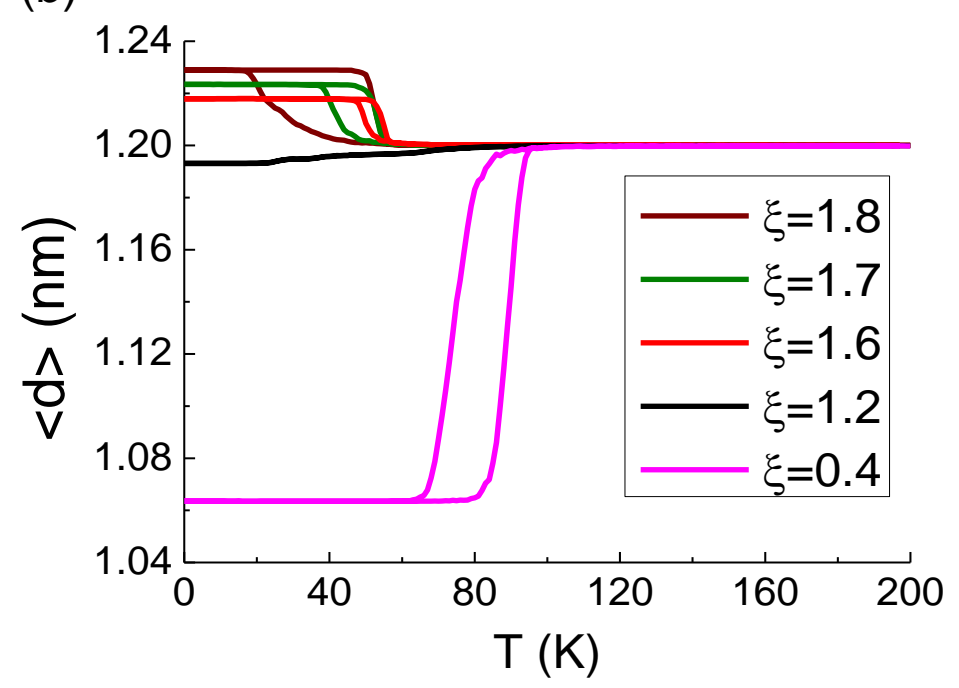

Figure 8: (a) Thermal dependence of the HS fraction for strong frustration parameter values $\xi=$ $1.2,1.6,1.7,1.8,2$, showing incomplete spin transitions (b) thermal-dependence of the corresponding average $n n$ distances. Only three values are obtained for the HS residual fractions $\left(n_{H S}\right.$ values at $\left.0 \mathrm{~K}\right): 1 / 3$,

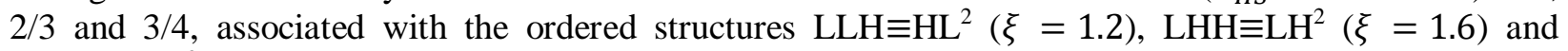
$\mathrm{LHHH} \equiv \mathrm{LH}^{3}(\xi=2)$. Remark the existence of the re-appearance of a "first order transition" for strong elastic frustrations, $\xi=1.6-2$. The parameter values are the same as those of Fig. 2.

Figures 9, presents the electroelastic configuration of the chain at $T=1 \mathrm{~K}$, corresponding respectively to $\xi=1.2$, and 1.6 of Fig. 8. The electronic configuration is given by the information of the spin state of each site (blue=LS and red=HS) and the information about the elastic configuration is obtained through the variation of the consecutive nn distances along the chain direction $(x)$. The latter is denoted $d_{x}$ and is given by $d_{x}=\left|\vec{r}_{i+1}-\vec{r}_{i}\right|$. Thus for $\xi=1.2$, the lowtemperature phase is made of a well organized LLH $\left(\mathrm{L}^{2} \mathrm{H}\right)$ structure, with the presence of some 
anti-phase boundaries which separate the two equivalent structures $\mathrm{L}^{2} \mathrm{H}$ and $\mathrm{HL}^{2}$. Interestingly, the spatial-dependence of the corresponding $n$ distances along the chain axis gives a periodic modulated structure, perturbed at some points by the presence of these phase boundaries. The modulation of the structure contrasts with that of HL phase of Fig. 3, for which $d_{x}$ remains uniform between two consecutive anti-phase boundaries.

\section{(a)}

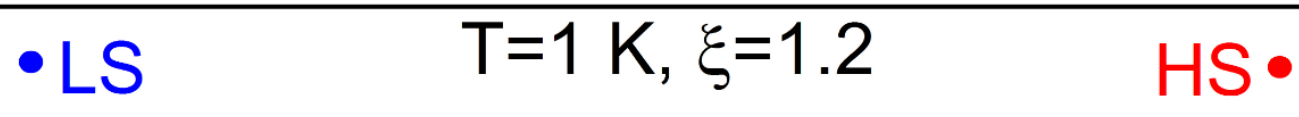

$$
\mathrm{T}=1 \mathrm{~K}, \xi=1.2
$$

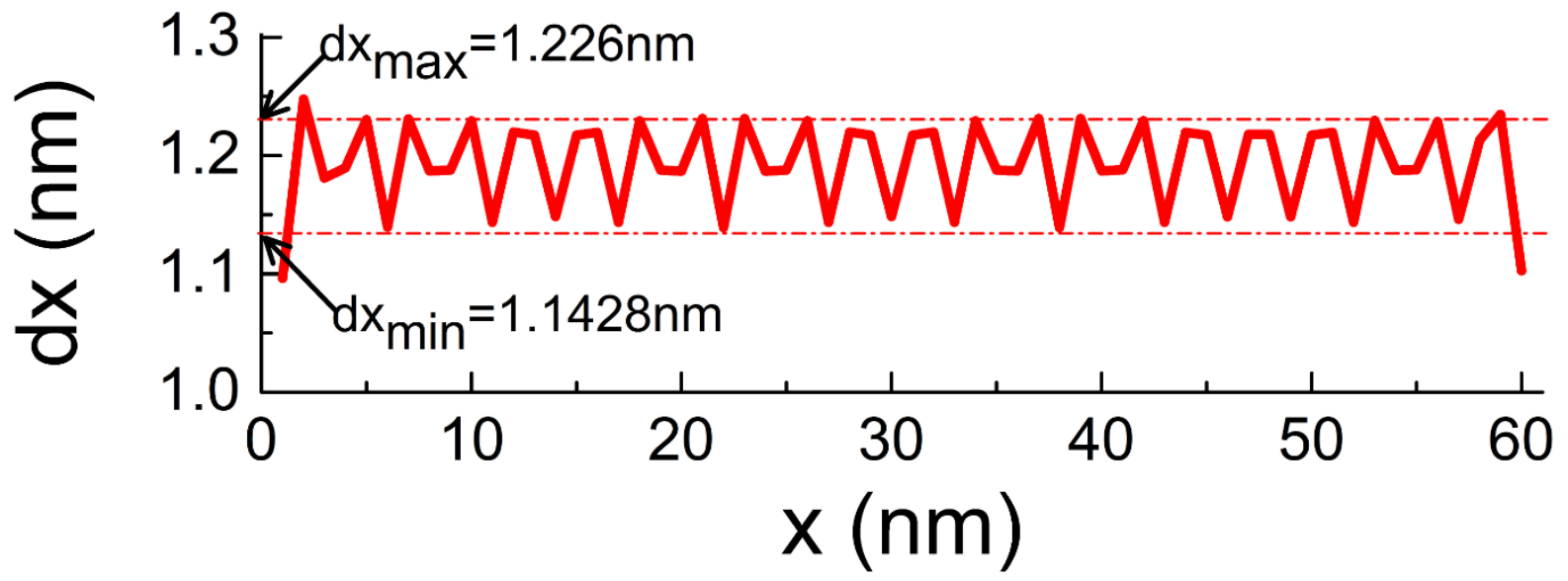


(b)

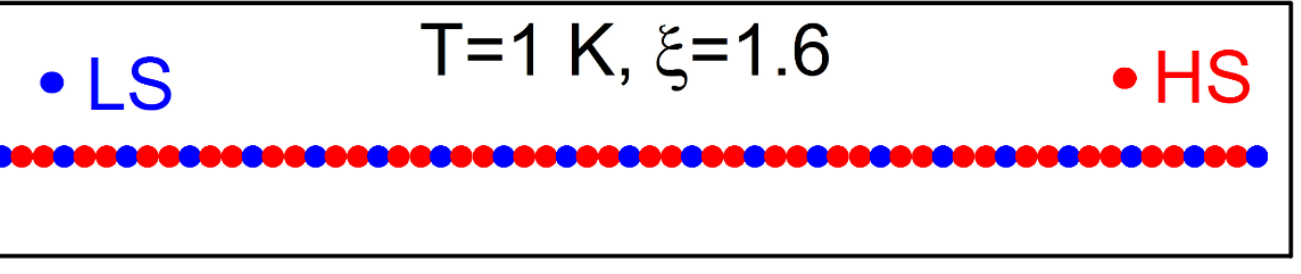

\section{$\mathrm{T}=1 \mathrm{~K}, \xi=1.6$}

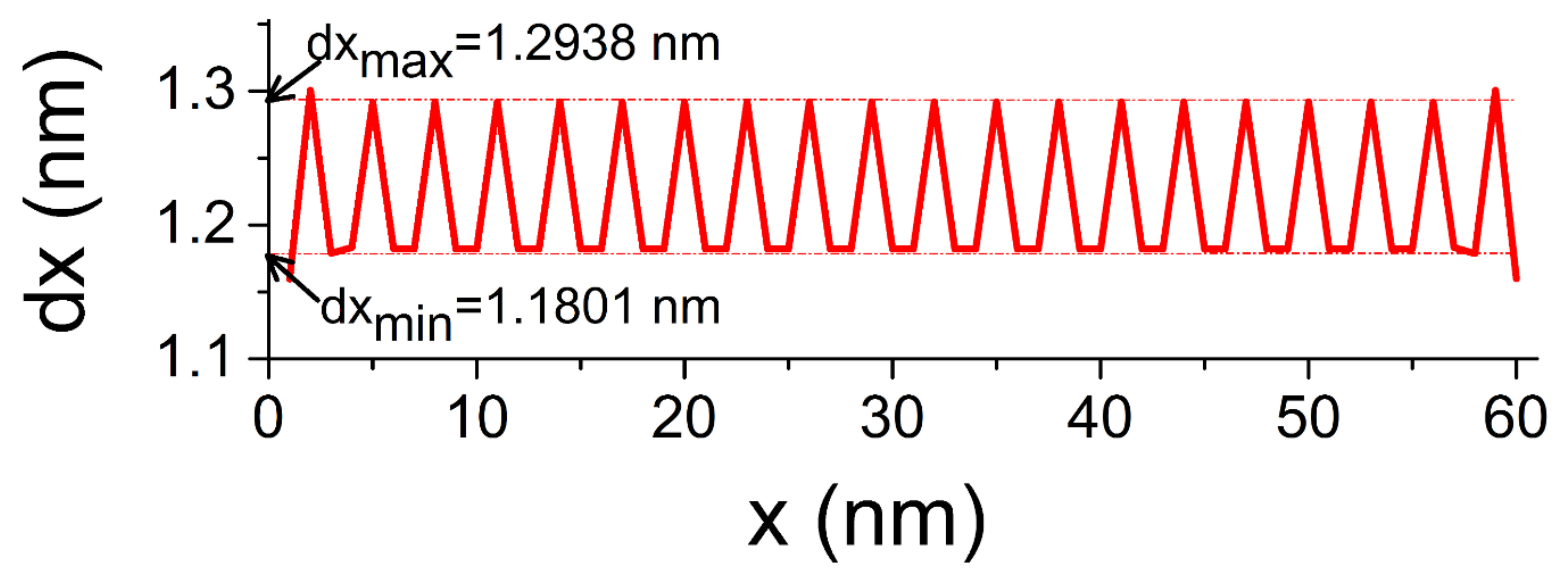

Figure 9: Electronic and elastic configurations of the chain corresponding to the low-temperature phase of Fig. 8 for (a) $\xi=1.2$ at $T=1 \mathrm{~K}$ and (b) $\xi=1.6$ at $T=1 \mathrm{~K}$. For $\xi=1.2$, two types of equivalent orders $\mathrm{L}^{2} \mathrm{H}$ and $\mathrm{HL}^{2}$ are obtained with the presence of anti-phase boundaries. The associated nn distance modulates spatially along the chain. For $\xi=1.6$, a perfect $\mathrm{LH}^{2}$ structure is obtained with a modulation of the lattice parameter.

Similarly, Figure 9b illustrates the configuration of the low-temperature state of the partial transition obtained in Fig. 8 for $\xi=1.6$. Now, the HS fraction is $\frac{2}{3}$, and a perfect $\mathrm{LHH}\left(\mathrm{LH}^{2}\right)$ ordered state is obtained. Again, the spatial distribution of the nn distances shows a nice periodic modulated structure, in qualitative very good agreement with several experimental results $22-23,34-$ ${ }^{37}$ reported in the literature of SCO materials.

Obviously, the state $\mathrm{LH}^{2}$ already started to appear around $T=50 \mathrm{~K}$ for $\xi=1.6$, as revealed by the presence of a plateau at $n_{H S}=\frac{2}{3}$ in the black curve of Fig. 8a. However, the presence of thermal fluctuations, introduce entropic effects in the structure, which renders much more difficulty in distinguishing $\mathrm{LH}^{2}$ and $\mathrm{H}^{2} \mathrm{~L}$ states. Thus, at $T=25 \mathrm{~K}$, for example, one expects a 
between $\mathrm{LH}^{2}, \mathrm{H}^{2} \mathrm{~L}$ on the one hand and $\mathrm{HL}^{2}, \mathrm{~L}^{2} \mathrm{H}$ form the other hand and then one will deal with an interplay between the complexity and the disorder.

Obviously, the phase $\mathrm{LH}^{2}$ already started to appear around $\mathrm{T}=50 \mathrm{~K}$ for $\xi=1.6$, as revealed by the presence of a plateau at $n_{H S}=\frac{2}{3}$ in the black curve of Fig. 6a. However, the presence of thermal fluctuations introduces entropic effects in the structure, which renders much more difficulty in distinguishing $\mathrm{LH}^{2}$ and $\mathrm{HL}^{2}$ states. Thus at $T=25 \mathrm{~K}$, for example, one expects a transition between $\mathrm{LH}^{2}, \mathrm{HL}^{2}$ on the one hand and $\mathrm{LH}^{2}, \mathrm{~L}^{2} \mathrm{H}$ from the other hand. One will deal then with the complexity, self-organization ${ }^{69}$ and disorder.

To ensure that we have not skipped any intermediate configuration, we conducted intensive MC simulations at very low-temperature, in which we calculate the HS fraction as a function of $\xi$, used here as a control parameter. The results are presented in Fig. 10. A multi-step transition is obtained with hysteresis, revealing the $\xi$ values at which the lattice undergoes a structural instability. An inspection of two lattice snapshots for the two selected values $\xi=1.25$ and $\xi=$ 1.8, lead to perfectly organized states, $\mathrm{L}^{2} \mathrm{H}$ and $\mathrm{H}^{2} \mathrm{~L}$, as those of Fig. 8. Obviously, changing the temperature will change the diagram $n_{H S}(\xi)$ of Fig. 10, due to the already discussed entropic effects.

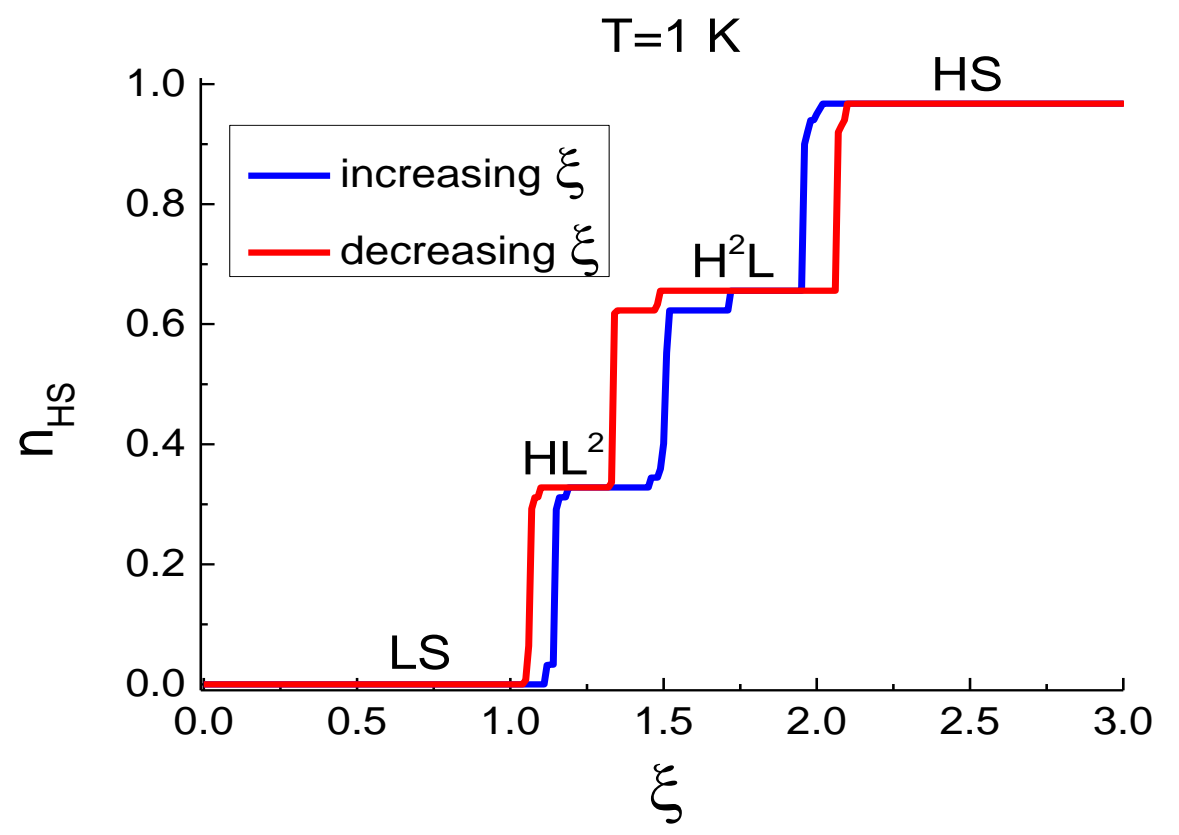


Figure 10: frustration-dependence of the HS fraction at $1 \mathrm{~K}$, showing a multi-step behavior. The transition from the LS to the HS state goes through the stabilization of two ordered intermediate states, namely $\mathrm{HL}^{2}$ and $\mathrm{LH}^{2}$, whose spatial structures are given in Fig. 8. The model parameters are the same as those of Fig. 2 .

The emergence of the $\mathrm{HL}, \mathrm{L}^{2} \mathrm{H}$ and $\mathrm{H}^{2} \mathrm{~L}$ structures are clearly intrinsic to the one-dimensional structure of the chain. For example in the two-dimensional version of this model, that we studied recently ${ }^{19}$, we found complex organized structures in partial SCO transitions, among which the antiferro-like structure and more complicated ones like $\mathrm{H}^{4} \mathrm{~L}$, whose spatial organization recalled Penrose's patterns.

So, to get more insights about the physical reasons for emergence of these particular, $\mathrm{HL}, \mathrm{HL}^{2}$, $\mathrm{H}^{2} \mathrm{~L}$, states, we calculated analytically the total relaxed energies of these three phases. In the following, we attempt an analytical determination of the relaxed nn distances for these three configurations, from which we derive the mechanically relaxed elastic energy. Let's start with the modulated structure $\mathrm{HL}^{2}$ (=HLLHLLHLL...). Here one sees that the structure consists of a periodic series of HS-LS, LS-LS, LS-HS etc. As a consequence, the nn can be only LS-LS or HSLS. Let us denote by $x$ the non-relaxed LS-LS nn distance and by $y$ that of the nn HS-LS distance. The total energy, which includes the electronic and (nn and nnn) elastic contributions writes (see SM for the details).

$$
\begin{gathered}
E_{L^{2} H}=\frac{-N}{3}\left(\Delta_{0}-k T \ln g\right)+\frac{N A_{0}}{3}\left[\left(x-R_{0}^{L L}\right)^{2}+2\left(y-R_{0}^{H L}\right)^{2}\right]+\frac{N B_{0}}{3}\left[2 \left(x+y-2 R_{0}^{L L}-\right.\right. \\
\left.\left.2 \delta_{R} \xi\right)^{2}+\left(2 y-2 R_{0}^{H L}-\delta_{R} \xi\right)^{2}\right]
\end{gathered}
$$

The relaxed LS-LS and HS-LS distances are determined by minimizing $E_{L^{2} H}$ with respect to $x$ and $y$, i.e., $\frac{\partial E_{L^{2} H}}{\partial x}=0$ and $\frac{\partial E_{L^{2} H}}{\partial y}=0$, which leads to the following relaxed nn distances,

$$
\begin{gathered}
x^{\text {relax }}=R_{L L}^{\text {relax }}=\frac{(k(k+3)-4) R_{0}^{L L}+2(k+4) R_{0}^{H L}+2 k \delta_{R} \xi}{k^{2}+5 k+4} \\
y^{\text {relax }}=R_{H L}^{\text {relax }}=\frac{k}{2} R_{0}^{L L}+2 R_{0}^{H L}+\delta_{R} \xi-\frac{k+2}{2} x^{\text {relax }}
\end{gathered}
$$

Replacing the parameters, $k=1, R_{0}^{L L}=1 \mathrm{~nm}, R_{0}^{H L}=1.2 \mathrm{~nm}$, and $\delta_{R}=0.2 \mathrm{~nm}$ by their numerical values, we evaluated in the case $\xi=1.2$, the following values for relaxed distances: $R_{L L}^{\text {relax }}=$ $1.148 \mathrm{~nm}$ and $R_{H L}^{\text {relax }}=1.218 \mathrm{~nm}$. The latter are in excellent agreement with the numerical results 
of MC simulations, shown in Fig. 8a, which gives a modulated structure with $R_{L L}^{\text {relax }}=1.1428 \mathrm{~nm}$ and $R_{H L}^{\text {relax }}=1.226 \mathrm{~nm}$.

Based on this success, we apply the same procedure to study the modulated structure $\mathrm{LH}^{2}$, (=LSLS-HS-LS-LS-HS...) whose total elastic energy writes

$E_{H^{2} L}=\frac{N}{3}\left(\Delta_{0}-k T \ln g\right)+\frac{N A_{0}}{3}\left[\left(x-R_{0}^{H H}\right)^{2}+2\left(y-R_{0}^{H L}\right)^{2}\right]+\frac{N B_{0}}{3}\left[2\left(x+y-2 R_{0}^{H L}-\right.\right.$ $\left.\left.\delta_{R} \xi\right)^{2}+\left(2 y-2 R_{0}^{H H}\right)^{2}\right] \quad(18)$

where $x$ is the distance between two nn HS-HS states and $y$ is that between two HS and LS nn sites. The minimization with respect to these two variables gives the following relaxed distances

$$
\begin{gathered}
x^{\text {relax }}=R_{H H}^{\text {relax }}=\frac{(k(k+3)-4) R_{0}^{H H}+2(k+4) R_{0}^{H L}+2(k+2) \delta_{R} \xi}{k^{2}+5 k+4} \\
y^{\text {relax }}=R_{H L}^{\text {relax }}=\frac{k}{2} R_{0}^{H H}+2 R_{0}^{H L}+\delta_{R} \xi-\frac{k+2}{2} x^{\text {relax }} \quad(19 \mathrm{~b})
\end{gathered}
$$

Here again, according to the model parameter values, which are the same as for the previous case, except for $\xi=1.6$, we evaluate $x^{\text {relax }}=R_{H H}^{\text {relax }}=1.292 \mathrm{~nm}$ and $y^{\text {relax }}=R_{H L}^{\text {relax }}=1.182 \mathrm{~nm}$. These values are here also in excellent agreement with those obtained from MC simulations, $R_{H H}^{\text {relax }}=1.2938 \mathrm{~nm}$ and $R_{H L}^{\text {relax }}=1.1801 \mathrm{~nm}$, shown in the snapshot of Fig. $8 \mathrm{~b}$.

Inserting the relaxed distances obtained in Eqs. (17) and (18) in their associated energies given in Eqs. (16) and (18), we immediately arrive at the relaxed energy densities, $\frac{E_{L^{2} H}^{\text {relax }}}{N}$ and $\frac{E_{H^{2} L}^{\text {relax }}}{N}$.

$$
\begin{aligned}
& \frac{E_{L_{2} H}^{r e l a x}}{N}=\frac{-1}{3}\left(\Delta_{0}-k T \ln g\right)+\frac{A_{0} \delta_{R}^{2}}{3}\left(\frac{329}{100} \xi^{2}-\frac{10}{19} \xi+\frac{3}{4}\right) \\
& \frac{E_{H^{2} L}^{r e l a x}}{N}=\frac{1}{3}\left(\Delta_{0}-k T \ln g\right)+\frac{A_{0} \delta_{R}^{2}}{3}\left(\frac{3}{5} \xi^{2}-\frac{9}{10} \xi+\frac{9}{8}\right)
\end{aligned}
$$

Figure 11a, summarizes the behavior of the total relaxed energies at $0 \mathrm{~K}$ of the five states, namely $\mathrm{LL}, \mathrm{L}^{2} \mathrm{H}, \mathrm{HL}$ and $\mathrm{H}^{2} \mathrm{~L}$ as the function of the frustration parameter, $\xi$. If we follow the lowest energy states by varying, $\xi$, we see clearly that from $\xi=0$ to $\xi=1.0031$, the $\operatorname{LS}\left(n_{H S}=0\right)$ state is fundamental and then a first transition to the $\mathrm{L}^{2} \mathrm{H}\left(n_{H S}=\frac{1}{3}\right)$ state occurs at $\xi=1.0031$. A second crossover is obtained for $\xi=1.5593$ at which the system switches to $\mathrm{H}^{2} \mathrm{~L}\left(n_{H S}=\frac{2}{3}\right)$ state, and then the last transition to the $\mathrm{HH}\left(n_{H S}=1\right)$ takes place at, $\xi=1.91$. Once again, these analytical predictions are in excellent agreement with MC data of Fig. 10. In addition, From Fig. 11a it is 
evident that in the interval $\xi=1.17-1.5$, several crossovers in close excited states occur between LL and HL, $\mathrm{H}^{2} \mathrm{~L}$ and HL, as well as between LL and $\mathrm{H}^{2} \mathrm{~L}$. These transitions can be activated by a small temperature change, although the latter introduces a major impact on the energy level scheme, as shown in Fig. 11b.
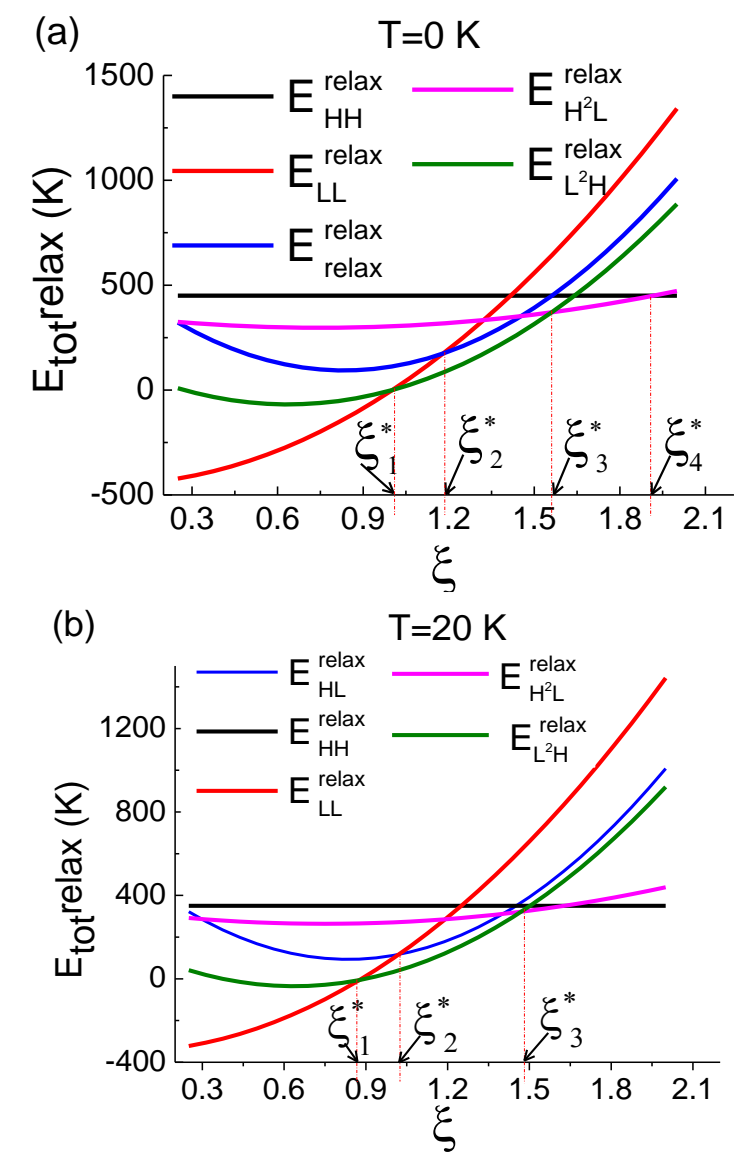

Figure 11: Calculated $\xi$-dependence of the total relaxed energies of the five states, $\mathrm{HH}, \mathrm{L}^{2} \mathrm{H}, \mathrm{HL}$, and $\mathrm{H}^{2} \mathrm{~L}$, (a) at $0 \mathrm{~K}$ and (b) at $20 \mathrm{~K}$. In the case a) four remarkable points located between $\xi_{1}^{*}=$ $1.0031, \xi_{4}^{*}=1.18191$ define the minimum energy pathway, along which the states among LL, $\mathrm{L}^{2} \mathrm{H}, \mathrm{H}^{2} \mathrm{~L}$ and $\mathrm{HH}$ will appear during the phase transitions. (b) The same reasoning is also used at $20 \mathrm{~K}$ where the transitions take place between at $20 \mathrm{~K}$ the $\xi_{1}$ to $\xi_{3}$ points are respectively equal $\xi_{1}^{*}=0.87$ and $\xi_{4}^{*}=1.47$. These results are in excellent agreement with MC data of Fig. 8.

\section{III.6 Evidence of reentrant spin transitions}

Among the interesting consequences of the elastic frustration, we mention the existence of nonmonotonous behavior of the thermal dependences of the HS fraction. Thus, Fig. 8b shows a clear evidence of a re-entrance phenomenon in the thermal behavior of the HS fraction when the 
frustration rate is increased. The re-entrance phenomenon is well known in physics of phase transition as a phenomenon (second order phase transition, for example) which exists at some values of a control parameter, then it disappears in some interval values and re-appears again beyond some threshold value. Examples in literature of re-entrant phenomena come from magnetism and ferroelectricity and have been intensively studied in the past ${ }^{70-72}$. In the present work, we remark that while for $\xi=0.4$, the chain displays a sharp transition with hysteresis although it is a kinetic one, between $\mathrm{HH}$ and LL states, the latter (i.e., the transition) transforms to gradual and incomplete between $\mathrm{HH}$ and $\mathrm{H}^{2} \mathrm{~L}$ states, which in turn converts to a mixed $\mathrm{L}^{2} \mathrm{H}-\mathrm{HL}$ state. Interestingly, by increasing $\xi$ values beyond the threshold, $\xi=1.2$, the thermal transition becomes sharper (case $\xi=1.6$ ) and the hysteresis re-appears again between $\mathrm{HH}$ and $\mathrm{H}^{2} \mathrm{~L}$ states $(\xi=1.6)$. The reappearance of the thermal transition is a signature of a re-entrant phase transition.

To understand this behavior one must analyze the dependence of the average value of the relaxed lattice parameter misfit between the $\mathrm{HH}$ and the $\mathrm{H}^{2} \mathrm{~L}$ states. As can be seen in Fig. $8 \mathrm{~b}$, the average value of the lattice parameter of the $\mathrm{H}^{2} \mathrm{~L}$ state at low-temperature exceeds the value of the nonfrustrated HS state, $R_{0}^{H S}$ for a certain value of $\xi \sim 1.25$. This value is exactly the one at which the misfit $x_{H H}^{\text {relax }}-x_{L L}^{\text {relax }}=\delta_{R}\left(1-\frac{4 \xi}{k+4}\right)$ changes its sign, i.e. for $\xi_{c}=\frac{k+4}{4}=1+\frac{A_{0}}{4 B_{0}} \quad(=1.25$, according to the used parameter values). For this special value, the relaxed nn distances of the $\mathrm{HH}$, LL and HL states are equal as shown in Fig. S3 of the SM. However, the elastic interaction energy

depends on the square of the lattice parameter misfit, i.e. $\left(x_{H H}^{\text {relax }}-x_{L L}^{\text {relax }}\right)^{2}$. As a result, the latter becomes minimum at $\xi=\xi_{c}$ and increases for $\xi>\xi_{c}$. This increase of the elastic energy due to frustration enhances the cooperativity of the system, which then recovers the thermal hysteretic behavior.

The present situation may take place at the surface of nanoparticles where the ligand field, the elastic constants and the equilibrium distances play in favor of the HS state, as does the $\xi$ parameter, here. Interestingly, re-entrant phase transitions have been reported very recently in SCO nanoparticles, studied by Mössbauer spectroscopy ${ }^{39,73}$ where the first-order character of the spin transition vanishes under some size and re-appears again below some a threshold nanoparticle size. It is then legitimate to ask whether the elastic frustration is at the origin of this phenomenon in such small systems

\section{Conclusion:}

1D spin-crossover solids are widely studied in the literature and they lead to a rich variety of behaviors, among them first-order, gradual, two-step, partial transitions etc. While already studied by $1 \mathrm{D}$ Ising-like models ${ }^{20,74-76}$ including only competing ferro- and antiferro- interactions which consist in ad-hoc interactions introduced to reproduce the multi-step character of some transitions. For our best knowledge, 1D SCO chains including elastic frustration have never been considered 
from the elastic point of view. So, we have designed in the present work a simple elastic model for 1D spin-crossover networks including an elastic frustration between nnn sites leading to antagonist equilibrium between nearest- and newt-nearest-neighbors. The model is solved using MC simulations performed on the spin states and the lattice positions. We have also developed an analytical method to predict the thermal behavior and the frustration dependence of the HS fraction and the nn relaxed distance, even for the case of modulated spin-state structures along the chain.

First of all, we demonstrated that the introduction of a frustration between nn and nnn equilibrium distances allows to stabilize intermediate states in the course of the thermal transition between the LS and the HS phases. According to the strength of the elastic frustration, we found that three

macroscopic values of the HS fraction, namely, $n_{H S}=\frac{1}{2}, \frac{1}{3}$ and $\frac{2}{3}$ can be obtained, corresponding to well organized antiferro-magnetic (HL), $\mathrm{L}^{2} \mathrm{H}$ and $\mathrm{H}^{2} \mathrm{~L}$ structures. An inspection of the spatial dependence of the lattice parameters in these three phases allowed to demonstrate that the HL state, except around the anti-phase boundaries, exhibits a uniform and constant lattice parameter, while $\mathrm{L}^{2} \mathrm{H}$ and $\mathrm{H}^{2} \mathrm{~L}$ patterns lead to modulated lattice parameter structures, which is commensurate with that of the spin state. The fact that this model gives, by changing only one parameter, almost all the observed thermal behaviors in SCO chains, is a strong indication of its relevance. Among the extensions of the present model, we mention the possibility to include the elastic frustration also in the HS state. Moreover, the frustration parameter itself may depend on temperature, thus following the difference of rigidities between the LS and HS states. The extension of the present model to include inter-chain elastic interactions surely deserves to be considered in a near future. Last but not least, we are currently extending this model to study the case of binuclear molecules which are known in literature to present thermally-induced two-step transition behavior, which has been only described in the past using Ising-like models including competing ferro- and anti-ferro like exchange interactions. This problem deserves to be studied using an adequate extension of the electro-elastic model.

\section{Acknowledgements}

This work is supported financially by the Université de Versailles Saint-Quentin, Université ParisSaclay, CNRS and the "Agence Nationale de la Recherche" (ANR project BISTA-MAT: ANR12-BS07-0030-01), which are acknowledged. R.T. thanks the government of Algeria for the research fellowship during his stay in GEMAC's laboratory. M. Sy thanks the Laboratoire of Excellence Charmmmat from the University Paris-Saclay for the post-doc fellowship. The authors warmly thank M. Yogendra Singh for his deep reading of the manuscript.

\section{ASSOCIATED CONTENT}


Supporting Information. Thermal dependence of the spatial distribution of the HS and LS states in the chain along the heating branch of a non-cooperative and cooperative 1D chain without elastic frustration. Thermal dependence of the fraction of neighboring HS-LS pairs. Mathematical developments on the spin-state dependence of the equilibrium distances for a frustrated system. Plot of the analytical linear $\xi$-dependence of the $\mathrm{HH}, \mathrm{LL}$ and $\mathrm{HL}$ relaxed lattice parameters at $0 \mathrm{~K}$. Analytical developments on the expressions of the transition temperatures in the case of two-step spin transitions.

\section{References:}

1. Phan, H.; Benjamin, S. M.; Steven, E.; Brooks, J. S.; Shatruk, M., Photomagnetic Response in Highly Conductive Iron(Ii) Spin-Crossover Complexes with Tenq Radicals. Angew. Chem., Int. Ed. Engl. 2015, 54, 823-827.

2. Ohkoshi, S.; Tokoro, H., Photomagnetism in Cyano-Bridged Bimetal Assemblies. Acc. Chem. Res. 2012, 45, 1749-1758.

3. Coronado, E.; Galán-Mascarós, J. R.; Monrabal-Capilla, M.; García-Martínez, J.; Pardo-Ibáñez, P., Bistable Spin-Crossover Nanoparticles Showing Magnetic Thermal Hysteresis near Room Temperature. Adv. Mater. 2007, 19, 1359-1361.

4. Kahn, O.; Jay-Martinez, C., Spin-Transition Polymers: From Molecular Materials toward Memory Devices. Science 1998, 279, 44-48.

5. Gütlich, P.; Hauser, A.; Spiering, H., Thermal and Optical Switching of Iron(Ii) Complexes. Angew. Chem., Int. Ed. Engl. 1994, 33, 2024-2054.

6. Gütlich, P.; Goodwin, H. A., Spin Crossover in Transition Metal Compounds I-Iii; Springer-Verlag Berlin Heidelberg: Berlin, Heidelberg, New York, 2004; Vol. 233, 234, 235.

7. Olguín, J.; Brooker, S., Spin Crossover Active Iron(Ii) Complexes of Selected PyrazolePyridine/Pyrazine Ligands. Coord. Chem. Rev. 2011, 255, 203-240.

8. Halcrow, M. A., Structure:Function Relationships in Molecular Spin-Crossover Complexes. Chem. Soc. Rev. 2011, 40, 4119-4142.

9. Prins, F.; Monrabal-Capilla, M.; Osorio, E. A.; Coronado, E.; van der Zant, H. S., RoomTemperature Electrical Addressing of a Bistable Spin-Crossover Molecular System. Adv. Mater. 2011, 23, $1545-1549$.

10. Gütlich, P.; Gaspar, A. B.; Garcia, Y., Spin State Switching in Iron Coordination Compounds. Beilstein J. Org. Chem. 2013, 9, 342-391.

11. Li, Z. Y.; Dai, J. W.; Shiota, Y.; Yoshizawa, K.; Kanegawa, S.; Sato, O., Multi-Step Spin Crossover Accompanied by Symmetry Breaking in an Fe(Iii) Complex: Crystallographic Evidence and Dft Studies. Chemistry 2013, 19, 12948-12952.

12. Halcrow, M. A., Spin-Crossover Materials: Properties and Applications John Wiley \& Sons Ltd, 2013.

13. Gallois, B.; Real, J. A.; Hauw, C.; Zarembowitch, J., Structural Changes Associated with the Spin Transition in Bis(Isothiocyanato)Bis(1,10-Phenanthroline)Iron: A Single-Crystal X-Ray Investigation. Inorg. Chem. 1990, 29, 1152-1158.

14. Milin, E.; Patinec, V.; Triki, S.; Bendeif, E.-E.; Pillet, S.; Marchivie, M.; Chastanet, G.; Boukheddaden, K., Elastic Frustration Triggering Photoinduced Hidden Hysteresis and Multistability in a 
Two-Dimensional Photoswitchable Hofmann-Like Spin-Crossover Metal-Organic Framework. Inorg. Chem. 2016, 55, 11652-11661.

15. Pittala, N.; Thétiot, F.; Triki, S.; Boukheddaden, K.; Chastanet, G.; Marchivie, M., Cooperative 1d Triazole-Based Spin Crossover Feii Material with Exceptional Mechanical Resilience. Chem. Mater. 2017, 29, 490-494.

16. Slimani, A.; Varret, F.; Boukheddaden, K.; Garrot, D.; Oubouchou, H.; Kaizaki, S., Velocity of the High-Spin Low-Spin Interface inside the Thermal Hysteresis Loop of a Spin-Crossover Crystal, Via Photothermal Control of the Interface Motion. Phys. Rev. Lett. 2013, 110, 087208-087213.

17. Koike, M.; Murakami, K.; Fujinami, T.; Nishi, K.; Matsumoto, N.; Sunatsuki, Y., Syntheses, Three Types of Hydrogen-Bonded Assembly Structures, and Magnetic Properties of [Feiii(Him)2(Hapen)] Y Solvent (Him=Imidazole, Hapen=N,N'-Bis(2Hydroxyacetophenylidene)Ethylenediamine, Y=Bph4-, Cf3so3-, Pf6-, Clo4-, and Bf4-). Inorg. Chim. Acta 2013, 399, 185-192.

18. Romstedt, H.; Hauser, A.; Spiering, H., High-Spin $\rightarrow$ Low-Spin Relaxation in the Two-Step Spincrossover Compound [Fe(Pic)3]Cl2etoh (Pic = 2-Picolylamine). J. Phys. Chem. Solids 1998, 59, 265275.

19. Paez-Espejo, M.; Sy, M.; Boukheddaden, K., Elastic Frustration Causing Two-Step and Multistep Transitions in Spin-Crossover Solids: Emergence of Complex Antiferroelastic Structures. J. Am. Chem. Soc. 2016, 138, 3202-3210.

20. Watanabe, H.; Tanaka, K.; Bréfuel, N.; Cailleau, H.; Létard, J.-F.; Ravy, S.; Fertey, P.; Nishino, M.; Miyashita, S.; Collet, E., Ordering Phenomena of High-Spin/Low-Spin States in Stepwise SpinCrossover Materials Described by the Annni Model. Phys. Rev. B 2016, 93, 014419-014430.

21. Ortega-Villar, N.; Muñoz, M.; Real, J., Symmetry Breaking in Iron(Ii) Spin-Crossover Molecular Crystals. Magnetochemistry 2016, 2, 16-37.

22. Trzop, E.; Zhang, D.; Piñeiro-Lopez, L.; Valverde-Muñoz, F. J.; Carmen Muñoz, M.; Palatinus, L.; Guerin, L.; Cailleau, H.; Real, J. A.; Collet, E., First Step Towards a Devil's Staircase in Spin-Crossover Materials. Angew. Chem., Int. Ed. Engl. 2016, 55, 8675-8679.

23. Murnaghan, K. D.; Carbonera, C.; Toupet, L.; Griffin, M.; Dirtu, M. M.; Desplanches, C.; Garcia, Y.; Collet, E.; Letard, J. F.; Morgan, G. G., Spin-State Ordering on One Sub-Lattice of a Mononuclear Iron(Iii) Spin Crossover Complex Exhibiting Liesst and Tiesst. Chemistry 2014, 20, 5613-5618.

24. Li, Z.-Y., et al., Direct Observation of Ordered High-Spin-Low-Spin Intermediate States of an Iron(Iii) Three-Step Spin-Crossover Complex. Angew. Chem., Int. Ed. Engl. 2016, 55, 5184-5189.

25. Hayami, S.; Nakaya, M.; Ohmagari, H.; Alao, A. S.; Nakamura, M.; Ohtani, R.; Yamaguchi, R.; Kuroda-Sowa, T.; Clegg, J. K., Spin-Crossover Behaviors in Solvated Cobalt(Ii) Compounds. Dalton Trans. 2015, 44, 9345-9348.

26. Bauer, W.; Pfaffeneder, T.; Achterhold, K.; Weber, B., Complete Two-Step Spin-Transition in a 1d Chain Iron(Ii) Complex with a 110-K Wide Intermediate Plateau. European Journal of Inorganic Chemistry 2011, 2011, 3183-3192.

27. Dirtu, M. M.; Schmit, F.; Naik, A. D.; Rusu, I.; Rotaru, A.; Rackwitz, S.; Wolny, J. A.; Schunemann, V.; Spinu, L.; Garcia, Y., Two-Step Spin Transition in a 1d Fe(Ii) 1,2,4-Triazole Chain Compound. Chemistry 2015, 21, 5843-5855.

28. Neville, S. M.; Leita, B. A.; Halder, G. J.; Kepert, C. J.; Moubaraki, B.; Letard, J. F.; Murray, K. S., Understanding the Two-Step Spin-Transition Phenomenon in Iron(Ii) 1d Chain Materials. Chemistry 2008, 14, 10123-10133.

29. Bousseksou, A.; Nasser, J.; Linares, J.; Boukheddaden, K.; Varret, F., Ising-Like Model for the Two-Step Spin-Crossover. J. Phys. I France 1992, 2, 1381-1403.

30. Bousseksou, A.; Varret, F.; Nasser, J., Ising-Like Model for the Two Step Spin-Crossover of Binuclear Molecules. J. Phys. I France 1993, 3, 1463-1473.

31. Boukheddaden, K.; Linares, J.; Codjovi, E.; Varret, F.; Niel, V.; Real, J. A., Dynamical Ising-Like Model for the Two-Step Spin-Crossover Systems. J. Appl. Phys. 2003, 93, 7103-7105. 
32. Nishino, M.; Boukheddaden, K.; Miyashita, S.; Varret, F., Arrhenius Monte Carlo Study of TwoStep Spin Crossover: Equilibrium and Relaxation Paths. Phys. Rev. B 2003, 68, 224402-224411.

33. Apetrei, A. M.; Boukheddaden, K.; Stancu, A., Dynamic Phase Transitions in the One-Dimensional Spin-Phonon Coupling Model. Phys. Rev. B 2013, 87, 014302-014307.

34. Pillet, S.; Bendeif, E.-E.; Bonnet, S.; Shepherd, H. J.; Guionneau, P., Multimetastability, Phototrapping, and Thermal Trapping of a Metastable Commensurate Superstructure in a Fe(Ii) SpinCrossover Compound. Phys. Rev. B 2012, 86, 064106-064117.

35. Collet, E., et al., Aperiodic Spin State Ordering of Bistable Molecules and Its Photoinduced Erasing. Phys. Rev. Lett. 2012, 109, 257206-257210.

36. Gaspar, A. B.; Ksenofontov, V.; Reiman, S.; Gutlich, P.; Thompson, A. L.; Goeta, A. E.; Munoz, M. C.; Real, J. A., Mossbauer Investigation of the Photoexcited Spin States and Crystal Structure Analysis of the Spin-Crossover Dinuclear Complex $[\{\mathrm{Fe}(\mathrm{Bt})(\mathrm{Ncs})(2)\}(2) \mathrm{Bpym}]\left(\mathrm{Bt}=2,2^{\prime}-\right.$ Bithiazoline, Bpym=2,2'Bipyrimidine). Chemistry 2006, 12, 9289-9298.

37. Fitzpatrick, A. J.; Trzop, E.; Muller-Bunz, H.; Dirtu, M. M.; Garcia, Y.; Collet, E.; Morgan, G. G., Electronic Vs. Structural Ordering in a Manganese(Iii) Spin Crossover Complex. Chem. Commun. 2015, $51,17540-17543$.

38. Chernyshov, D.; Hostettler, M.; Tornroos, K. W.; Burgi, H. B., Ordering Phenomena and Phase Transitions in a Spin-Crossover Compound-Uncovering the Nature of the Intermediate Phase of [Fe(2Pic)3]Cl2.Etoh. Angew. Chem., Int. Ed. Engl. 2003, 42, 3825-3830.

39. Peng, H.; Tricard, S.; Félix, G.; Molnár, G.; Nicolazzi, W.; Salmon, L.; Bousseksou, A., ReAppearance of Cooperativity in Ultra-Small Spin-Crossover $[\mathrm{Fe}(\mathrm{Pz})\{\mathrm{Ni}(\mathrm{Cn}) 4\}]$ Nanoparticles. Angew. Chem. 2014, 126, 11074-11078.

40. Miyashita, S.; Konishi, Y.; Nishino, M.; Tokoro, H.; Rikvold, P. A., Realization of the Mean-Field Universality Class in Spin-Crossover Materials. Phys. Rev. B 2008, 77, 014105-014116.

41. Nishino, M.; Boukheddaden, K.; Konishi, Y.; Miyashita, S., Simple Two-Dimensional Model for the Elastic Origin of Cooperativity among Spin States of Spin-Crossover Complexes. Phys. Rev. Lett. 2007, 98, 247203-247206.

42. Nishino, M.; Enachescu, C.; Miyashita, S.; Boukheddaden, K.; Varret, F., Intrinsic Effects of the Boundary Condition on Switching Processes in Effective Long-Range Interactions Originating from Local Structural Change. Phys. Rev. B 2010, 82, 020409-020412.

43. Stoleriu, L.; Chakraborty, P.; Hauser, A.; Stancu, A.; Enachescu, C., Thermal Hysteresis in SpinCrossover Compounds Studied within the Mechanoelastic Model and Its Potential Application to Nanoparticles. Phys. Rev. B 2011, 84, 134102-134110.

44. Enachescu, C.; Stoleriu, L.; Stancu, A.; Hauser, A., Model for Elastic Relaxation Phenomena in Finite 2d Hexagonal Molecular Lattices. Phys. Rev. Lett. 2009, 102, 257204-257207.

45. Nicolazzi, W.; Pillet, S.; Lecomte, C., Two-Variable Anharmonic Model for Spin-Crossover Solids: A Like-Spin Domains Interpretation. Phys. Rev. B 2008, 78, 174401-174412.

46. Nishino, M.; Enachescu, C.; Miyashita, S.; Rikvold, P. A.; Boukheddaden, K.; Varret, F., Macroscopic Nucleation Phenomena in Continuum Media with Long-Range Interactions. Sci. Rep. 2011, $1,162-167$.

47. Slimani, A.; Boukheddaden, K.; Varret, F.; Oubouchou, H.; Nishino, M.; Miyashita, S., Microscopic Spin-Distortion Model for Switchable Molecular Solids: Spatiotemporal Study of the Deformation Field and Local Stress at the Thermal Spin Transition. Phys. Rev. B 2013, 87, 014111-014121. 48. Boukheddaden, K., Anharmonic Model for Phonon-Induced First-Order Transition in 1-D SpinCrossover Solids. Prog Theor Phys 2004, 112, 205-217.

49. Konishi, Y.; Tokoro, H.; Nishino, M.; Miyashita, S., Monte Carlo Simulation of Pressure-Induced Phase Transitions in Spin-Crossover Materials. Phys. Rev. Lett. 2008, 100, 067206-067209.

50. Ye, H.-Z.; Sun, C.; Jiang, H., Monte-Carlo Simulations of Spin-Crossover Phenomena Based on a Vibronic Ising-Like Model with Realistic Parameters. Physical Chemistry Chemical Physics 2015, 17, 6801-6808. 
51. Varret, F.; Slimani, A.; Boukheddaden, K.; Chong, C.; Mishra, H.; Collet, E.; Haasnoot, J.; Pillet, S., The Propagation of the Thermal Spin Transition of [Fe(Btr)(2)(Ncs)(2)]Center Dot H2o Single Crystals, Observed by Optical Microscopy. New J. Chem. 2011, 35, 2333-2340.

52. $\quad$ Chong, C.; Mishra, H.; Boukheddaden, K.; Denise, S.; Bouchez, G.; Collet, E.; Ameline, J. C.; Naik, A. D.; Garcia, Y.; Varret, F., Electronic and Structural Aspects of Spin Transitions Observed by Optical Microscopy. The Case of [Fe(Ptz)(6)](Bf4)(2). J. Phys. Chem. B 2010, 114, 1975-1984.

53. Slimani, A.; Varret, F.; Boukheddaden, K.; Chong, C.; Mishra, H.; Haasnoot, J.; Pillet, S., Visualization and Quantitative Analysis of Spatiotemporal Behavior in a First-Order Thermal Spin Transition: A Stress-Driven Multiscale Process. Phys. Rev. B 2011, 84, 094442-094450.

54. Slimani, A.; Boukheddaden, K.; Varret, F.; Nishino, M.; Miyashita, S., Properties of the Low-Spin High-Spin Interface During the Relaxation of Spin-Crossover Materials, Investigated through an ElectroElastic Model. J. Chem. Phys. 2013, 139, 194706-194713.

55. Slimani, A.; Boukheddaden, K.; Yamashita, K., Thermal Spin Transition of Circularly Shaped Nanoparticles in a Core-Shell Structure Investigated with an Electroelastic Model. Phys. Rev. B 2014, 89, 214109-214116.

56. Kamel, B.; Aurélien, B.-R., Towards the Elastic Properties of 3d Spin-Crossover Thin Films: Evidence of Buckling Effects. Europhys. Lett. 2013, 103, 26005-26010.

57. Sy, M.; Varret, F.; Boukheddaden, K.; Bouchez, G.; Marrot, J.; Kawata, S.; Kaizaki, S., StructureDriven Orientation of the High-Spin-Low-Spin Interface in a Spin-Crossover Single Crystal. Angew. Chem., Int. Ed. Engl. 2014, 53, 7539-7542.

58. Slimani, A.; Boukheddaden, K.; Yamashita, K., Effect of Intermolecular Interactions on the Nucleation, Growth, and Propagation of Like-Spin Domains in Spin-Crossover Materials. Phys. Rev. B 2015, 92, 014111-014121.

59. Boukheddaden, K.; Slimani, A.; Sy, M.; Varret, F.; Oubouchou, H.; Traiche, R., Physical Properties of 2d Spin-Crossover Solids from an Elastonic Description: Effect of Shape, Size and Spin-Distortion Interactions. In Magnetic Structures of $2 d$ and $3 d$ Nanoparticles - Properties and Applications, CRC Press, 2016 ed.; Jean-Claude Levy (Université Paris-Diderot, F., Ed. Pan Stanford Publishing: Pan Stanford Publishing, 2016; p 70.

60. Boukheddaden, K.; Traiche, R.; Oubouchou, H.; Linares, J., Multistep Relaxations in a SpinCrossover Lattice with Defect: A Spatiotemporal Study of the Domain Propagation. Magnetochemistry 2016, 2, 17-27.

61. Sorai, M., Heat Capacity Studies of Spin Crossover Systems. In Spin Crossover in Transition Metal Compounds Iii, Springer Berlin Heidelberg: Berlin, Heidelberg, 2004; pp 153-170.

62. Itoi, M.; Maurin, I.; Varret, F.; Frye, F. A.; Talham, D. R.; Chernyshov, D.; Boukheddaden, K., When Local Deformations Trigger Lattice Instability: Flow Diagram Investigations for Photoinduced and Quenched Metastable States in a Prussian Blue Analog. Phys. Rev. B 2013, 88, 094104-094111.

63. Chong, C., et al., Metastable State of the Photomagnetic Prussian Blue Analog K_\{0.3\}Co[Fe(Cn)_\{6\}]_\{0.77\} $3.6 h \_\{2\} O$ Investigated by Various Techniques. Phys. Rev. B 2011, 84, $144102-144113$.

64. Imoto, K.; Takemura, M.; Tokoro, H.; Ohkoshi, S.-i., A Cyano-Bridged Vanadium-Niobium Bimetal Assembly Exhibiting a High Curie Temperature of 210 K. Eur. J. Inorg. Chem. 2012, 2012, 26492652.

65. Braun, O. M.; Kivshar, Y. S., Nonlinear Dynamics of the Frenkel-Kontorova Model. Physics Reports-Review Section of Physics Letters 1998, 306, 1-108.

66. Jung, J.; Bruchhäuser, F.; Feile, R.; Spiering, H.; Gütlich, P., The Cooperative Spin Transition in [Fe X Zn 1 - X (Ptz) 6 ](Bf 4 ) 2 : I. Elastic Properties - an Oriented Sample Rotation Study by Brillouin Spectroscopy. Zeitschrift für Physik B Condensed Matter 1996, 100, 517-522.

67. Spiering, H.; Boukheddaden, K.; Linares, J.; Varret, F., Total Free Energy of a Spin-Crossover Molecular System. Phys. Rev. B: Condens. Matter Mater. Phys. 2004, 70, 184106-184120.

68. Linares, J.; Jureschi, C.; Boukheddaden, K., Surface Effects Leading to Unusual Size Dependence of the Thermal Hysteresis Behavior in Spin-Crossover Nanoparticles. Magnetochemistry 2016, 2, 24-35. 
69. Chong, C.; Varret, F.; Boukheddaden, K., Evolution of Self-Organized Spin Domains under Light in Single-Crystalline [Fe(Ptz)6](Bf4)2. Phys. Rev. B 2010, 81, 014104-014113.

70. Boukheddaden, K.; Varret, F., Vibronic Theory of Electric Hysteresis in "Bistable" Mixed-Valence Molecular Salts. Chemical Physics 1997, 216, 373-389.

71. Blume, M.; Emery, V. J.; Griffiths, R. B., Ising Model for the \$LEnsuremath\{Lambda\}\$ Transition and Phase Separation in $\$\{\backslash \text { Mathrm }\{\mathrm{He}\}\}^{\wedge}\{3\} \$-\$\{\text { Mathrm }\{\mathrm{He}\}\}^{\wedge}\{4\} \$$ Mixtures. Physical Review A 1971, 4, 1071-1077.

72. Guerroudj, S.; Caballero, R.; De Zela, F.; Cătălin Maricel, J.; Linares, J.; Boukheddaden, K., Monte Carlo - Metropolis Investigations of Shape and Matrix Effects in 2d and 3d Spin-Crossover Nanoparticles. 2016, 738, 012068-012074.

73. Boukheddaden, K.; Linares, J.; Spiering, H.; Varret, F., One-Dimensional Ising-Like Systems: An Analytical Investigation of the Static and Dynamic Properties, Applied to Spin-Crossover Relaxation. Eur. Phys. J. B 2000, 15, 317-326.

74. Boukheddaden, K.; Linares, J.; Tanasa, R.; Chong, C., Theoretical Investigations on an Axial Next Nearest Neighbour Ising-Like Model for Spin Crossover Solids: One- and Two-Step Spin Transitions. $J$. Phys.: Condens. Matter 2007, 19, 106201-106212.

75. Boukheddaden, K.; Miyashita, S.; Nishino, M., Elastic Interaction among Transition Metals in OneDimensional Spin-Crossover Solids. Phys. Rev. B 2007, 75, 094112-094121.

76. Chiruta, D.; Jureschi, C.-M.; Linares, J.; Dahoo, P. R.; Garcia, Y.; Rotaru, A., On the Origin of Multi-Step Spin Transition Behaviour in 1d Nanoparticles. Eur. Phys. J. B 2015, 88, 233-237. 